\title{
EFFECTS OF LAND USE CHANGES AND LAND VEGETATION ON WATER BIRDS AND WADERS DIVERSITY OF ANZALI, ALAGOL, ALMAGOL AND AJIGOL INTERNATIONAL WETLANDS
}

\author{
Narjes Nazari ${ }^{1}$ \\ Bahman Shams Esfandiari ${ }^{2}$ \\ Javad Varvani ${ }^{3}$ \\ Abas Ahmadi ${ }^{4}$ \\ Hamid Toranjzar ${ }^{5}$
}

\begin{abstract}
The purpose of this study was to determine the diversity of wintering water birds and waders in Alagol, Almagol, Ajigol and Anzali wetlands and the impact of land use changes on the distribution of these wetlands.Materials and Methods: ETM + satellite images after atmospheric and geometrical correction were used to evaluate the changes. The NDVI index identified the overall vegetation cover of the wetlands on and out of the coast. Then, dominant plants and representative of wetland
\end{abstract}

status were identified and evaluated by unsupervised classification. The diversity of water birds and waders was assessed using Total Count Method. Species diversity index, species uniformity and interspecific similarity were calculated and reported.Results: The catchment area of the studied wetland has decreased significantly during the last 10 years and the type of vegetation and area of the catchment area has increased toward nutritional state. The highest number of migratory water

\footnotetext{
${ }^{1} \mathrm{PhD}$, Department of Environment, Islamic Azad University, Falagh Branch, Iran.

Email: Mersa.s.t.1366@gmail.com.

${ }^{2} \mathrm{PhD}$, Department of Environment, Islamic Azad University, Falagh Branch, Iran.

Email: submitarticlejournal@gmail.com (Corresponding Author)

${ }^{3}$ Associate Professor, Department of Environment, Arak Branch, Islamic Azad

University, Arak. Iran. Email: Varvani_55@yahoo.com

${ }^{4}$ Faculty member, Department of Environment, Arak Branch, Islamic Azad University, Arak. Iran. Email: Abahmadi@gmail.com.

${ }^{5}$ Faculty member, Department of Environment, Arak Branch, Islamic Azad University, Arak. Iran. Email: h-toranjzar@iau-arak.ac.ir.
} 
birds belonged to the duckweed, with an average of $65.99 \%$ of the total migratory bird population belonged to this species, especially the water feeding ducks.Conclusion: Due to the lack of legal compliance with the wetland and the increasing use of agricultural and human-made land uses, this changing and growing land use will have adverse effects on the wetland ecosystem.

Keywords: Alagol, Almagol, Ajigol, Anzali, Wetland.

\section{Introduction}

Wetlands are among the richest ecosystems that are important for biodiversity conservation (Amat and Green, 2010). However, it may be argued that wetlands are more neglected than other natural habitats. The most important reasons in this regard are land conversion and invasion of wetlands due to the inefficiency of existing laws in protecting wetlands, and the decline in the quality of existing wetlands is due to various causes, such as the entering of other types of pollution (Ballan et al., 2002). Therefore, it is important to evaluate the changing trends of wetland use and the continuous monitoring of surrounding lands. Accurate and timely understanding of changes in land surface effects is important for better understanding of human communication and interactions with natural phenomena in order to properly manage and utilize resources at the spatial and temporal scales (Gallego, 2004). With 250 wetlands, Iran is ranked first in the importance of wetland systems in the Middle East, among these wetlands, Anzali, Alagol, Almagol and Ajigul International Wetlands that are registered in the Ramsar Convention and it is very important in many aspects, It is also observed that the conversion of land and the introduction of sediments and types of pollutants and nutrients into the wetland have reduced the natural life of these valuable aquatic ecosystems, So that, if the current situation continues, these wetlands will disappear much sooner than their natural destiny (Ghorbani, Taghipour, Mahmoudzadeh, 2012). Birds are one of the most important indicators of wetland habitat suitability. Studying and comparing the density and diversity of birds in a few years in a habitat can well represent the desirable or unfavorable habitat quality and other necessary conditions for each 
species (Brown and Dinsmore, 1986). Therefore, the study of the changes in the diversity and population of aquatic birds in different years can be considered as a biomarker of health status or the presence of threats in wetland ecosystems. Therefore, the purpose of this study was to investigate the impact of land use changes and land vegetation cover on water birds and wader's diversity of Anzali, Alagol, Almagol and Ajigul wetlands.

\section{Materials and Methods}

Study area, Alagol wetlands (2500 hectares), Almagol (207 hectares), Ajigol (320 hectares) near IranTurkmenistan border in Golestan province and Anzali wetland (15000 hectares) in geographical position. $37^{\circ} 25^{\prime}$ to $37^{\circ} 30^{\prime}$ north latitude and $49^{\circ} 25^{\prime}$ to $49^{\circ} 30^{\prime}$ east longitude on the southwest coast of the Caspian Sea in Gilan province, Iran.

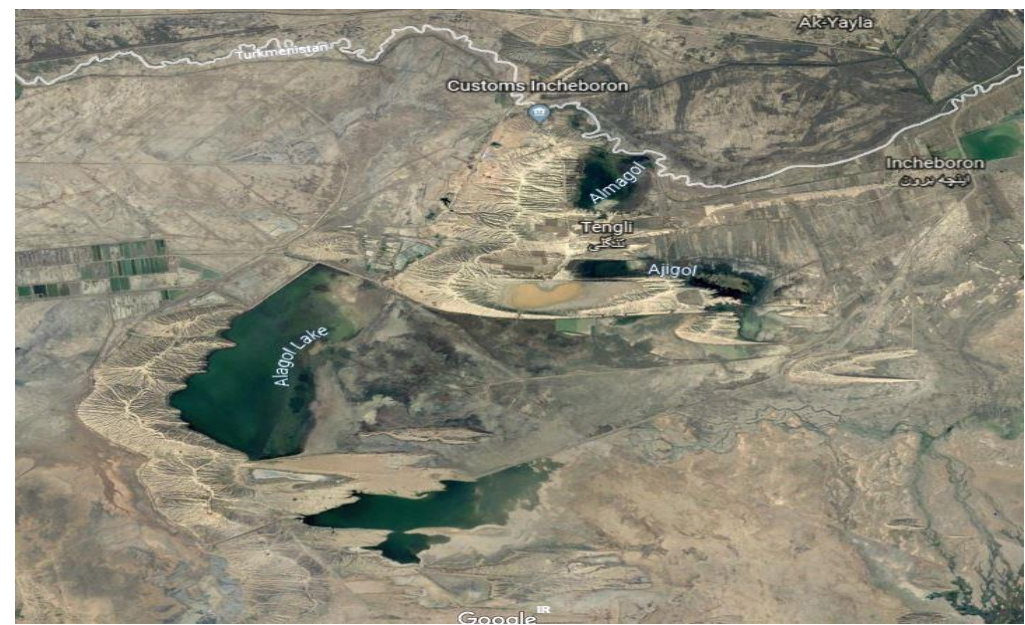

Figure 1: Location of Almagol, Ajigul and Alagol Wetlands

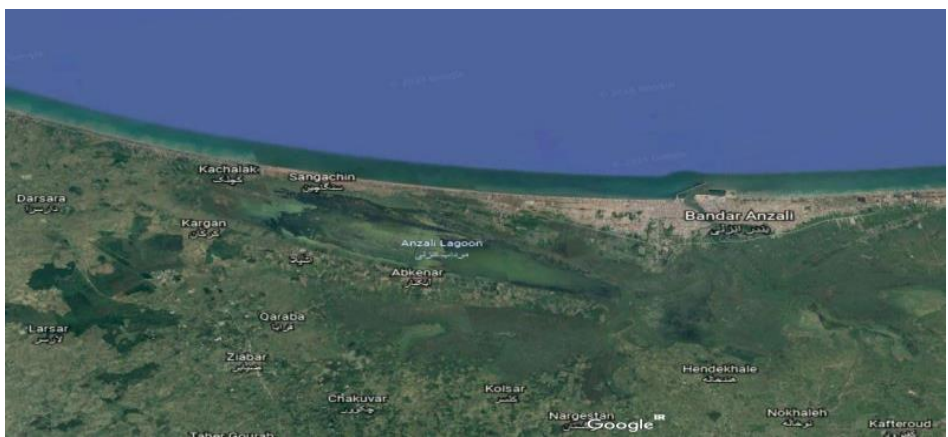

Figure 2: Anzali Wetland Location 


\section{Land use Changes}

For the purpose of investigating the changes, ETM + multi-temporal satellite images were selected in the dates listed in Table 1, the Anzali, Alagol, Almagol and Ajigul International Wetlands area, which were available.

Table 1: Sources of information from the types of satellite image

\begin{tabular}{|c|c|c|}
\hline Resolution & Sensor & Year \\
\hline 30 meters & ETM+ & 1987 \\
\hline 30 meters & ETM+ & 2000 \\
\hline 30 meters & ETM+ & 2005 \\
\hline 30 meters & ETM+ & 2010 \\
\hline 30 meters & ETM+ & 2019 \\
\hline
\end{tabular}

Data conversion and satellite data processing were performed for the use of satellite numeral information in the ILWIS software environment. After preparing multi-temporal images of the area, all the satellite images were subjected to radiometric, geometric corrections and normalization techniques in the pre-processing phase. In this study, the dark pixels value method was used, using ETM + 7 band, where the water and shadow in this band have zero DN values, the pixel digits of bands 1 to 5 and 7 of the region were positioned on $\mathrm{X}$ and $\mathrm{Y}$ axes relative to each other, and by using least squares method, the amount of atmospheric error (distance from origin) was deducted from the whole band data from 1 to 5 . The pixel-to-pixel split images range from -255 to +255 for ETM + images. Next, the classification of satellite images was used to attribute the numeral values in the image to groups with homogeneous characteristics, with the aim of distinguishing different objects or phenomena from each other. Maximum likelihood classification was performed using the following formula (Seaby and Henderson, 2007).

$$
L_{k}=P(k / x)=P(k) * P(x / k) / \sum P(i) * P(x / i)
$$


The following relation was also

used to separate image categories

(Seaby and Henderson, 2007).

$$
L_{k}(x)=\frac{1}{(2 \pi)^{n / 2}\left|\sum_{k}\right| 1 / 2} \exp \left\{-\frac{1}{2}\left(x-\mu_{k}\right) \cdot \Sigma^{-1} \cdot\left(x-\mu_{k}\right)^{t}\right\}
$$

In addition to using the automatic method for separating water and land boundaries, numeral and visual interpretation was used according to the Ozesmi and Bauer method (Ozesmi and Bauer, 2002).

\section{Investigation of Vegetation}

\section{Distribution}

For this purpose, the 6 bands TM sensor Landsat satellite 5 used from 2009 to 2019 for NVIDA imaging. Also, field survey and sampling of plants, index of each wetland, identification and dominance of plants in the area were determined. This information was used to explain unsupervised classification using the ISOCLUST module. In order to determine the dominance of plants in the area, a point sampling and evaluation method along transect from proximity to drought was considered. The initial length of each transect was assumed to be one kilometer, which increased or decreased with respect to topographic conditions. Also, the number of transects, their path and location of sampling points based on environmental changes along the slope were determined by satellite imagery and initial field visit. In areas where it was not possible to continue transect, additional points were considered. In sum, the position of these points was entered into the GPS device by DNRGarmin software. According to the vegetation layers, three circular plots with radius of 1, 5 and $9 \mathrm{~m}$ were selected for land cover, bushes, seedlings and trees, respectively. By selecting the sampling location with the conditions listed above, circular plots of the same sizes as estimated for each of the layers estimated to estimate the abundance of each, begin first with the ground cover layer (if any) and by observing the specified plots. The abundance of plants for each species and layers in the plot was evaluated by percentage of cover. 

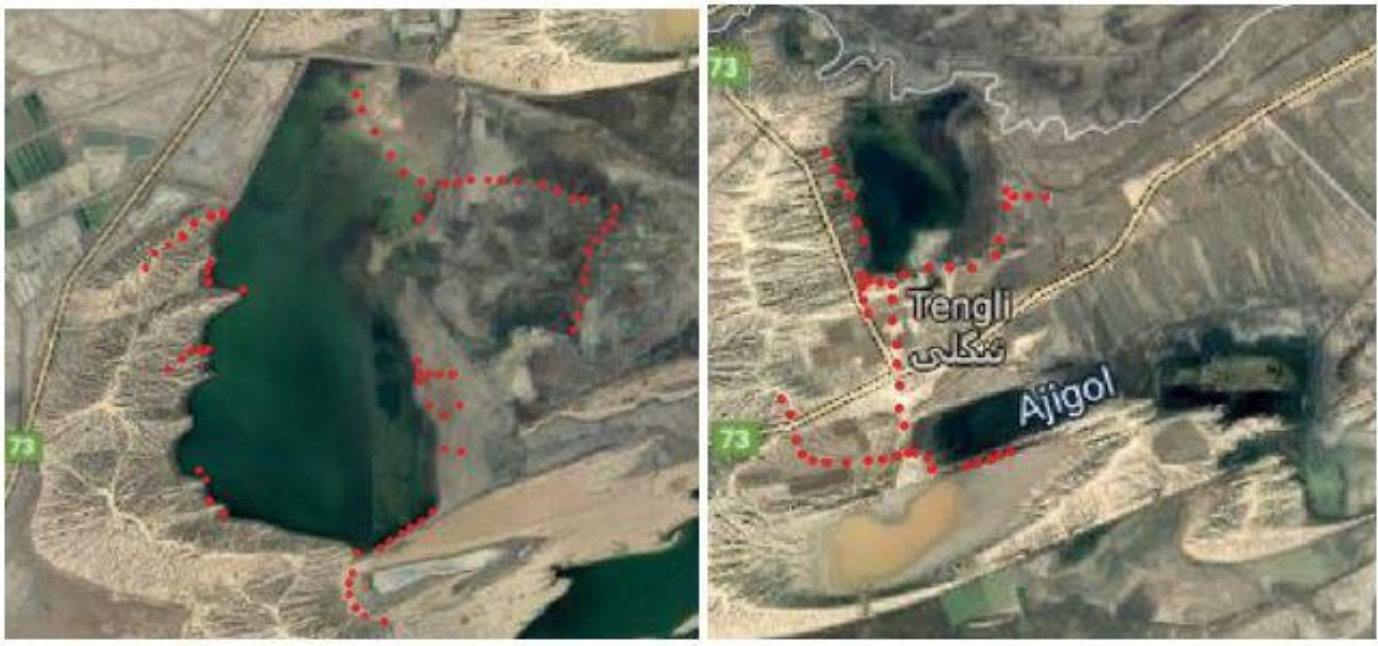

Figure 3: Location of sampling points, number of transects and their path in Almagol, Ajigol and Alagol wetlands

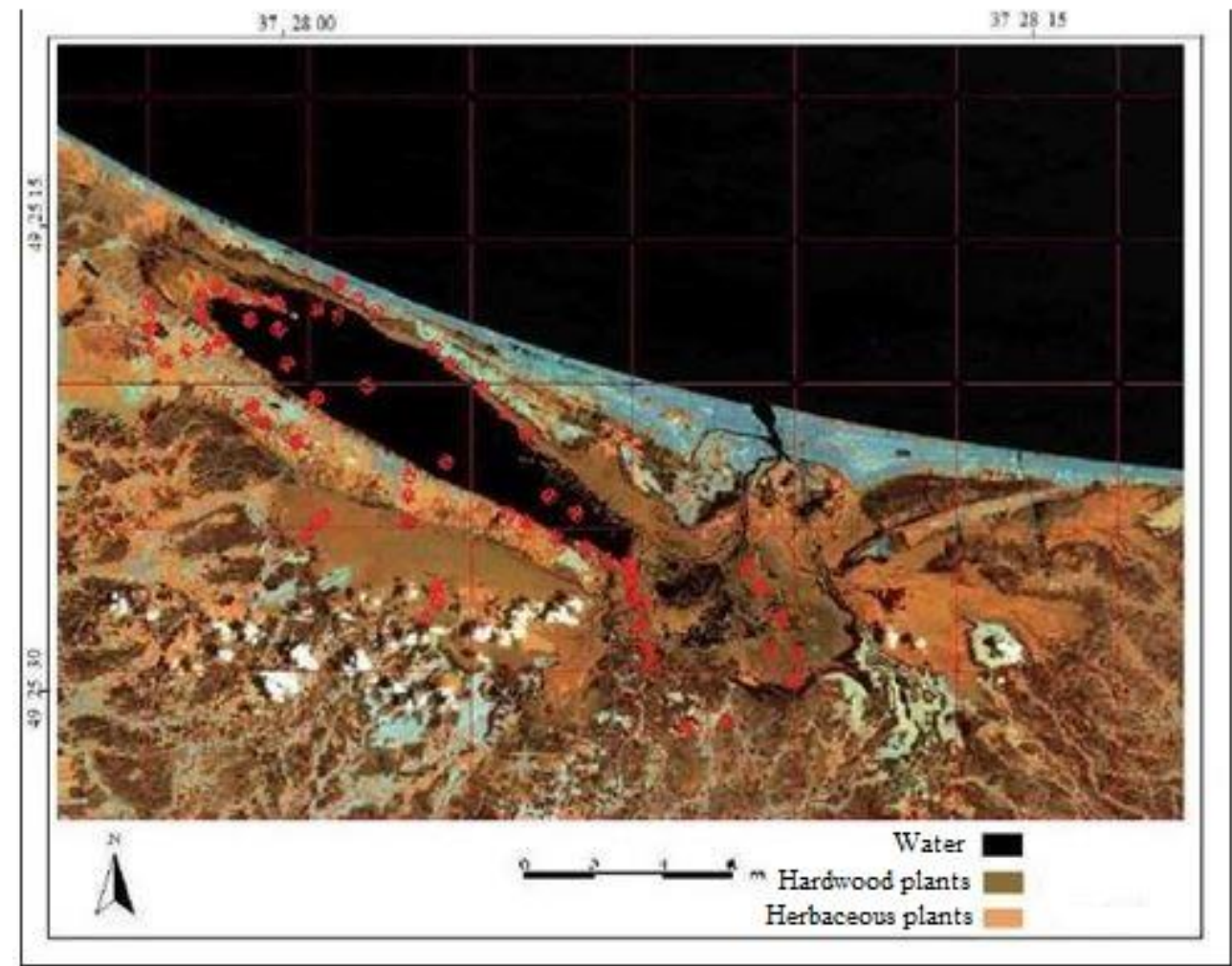


Figure 4: Location of sampling points, number of transects and their path in

Anzali Wetland

\section{Species diversity of water birds and waders}

In recent years, the wetlands under study from January to February for four days each year for water birds and waders counted by experts from the General Directorate of Environmental Protection of Guilan and Golestan. To identify birds in the field from the Wild Birds Guidebook and Total Count method was used to count the birds (Camargo, 1993). In this study, the results of 8 years of census of aquatic birds in Anzali, Alagol, Almagol and Ajigol Wetlands were analyzed. To determine species diversity of water birds and waders in the studied years from Shannon-Wiener species diversity index and vice versa Simson index, To determine species richness of Margalph and Menhink indexes, to determine species uniformity of Pilo and Simpson and Moristas indices were used to determine the interspecific similarity of aquatic birds in different years and different parts of Anzali Wetland (Simpson, 1949; Morisita, 1959).

Finally, to link the relationship between wetland land use change and the distribution of birds by Pearson's correlation coefficient in SPSS program were used.

\section{Results}

Satellite imagery from 1987 to 2019 was first studied in order to study landslide trends of Alagol, Almagol and Ajigol wetlands. 


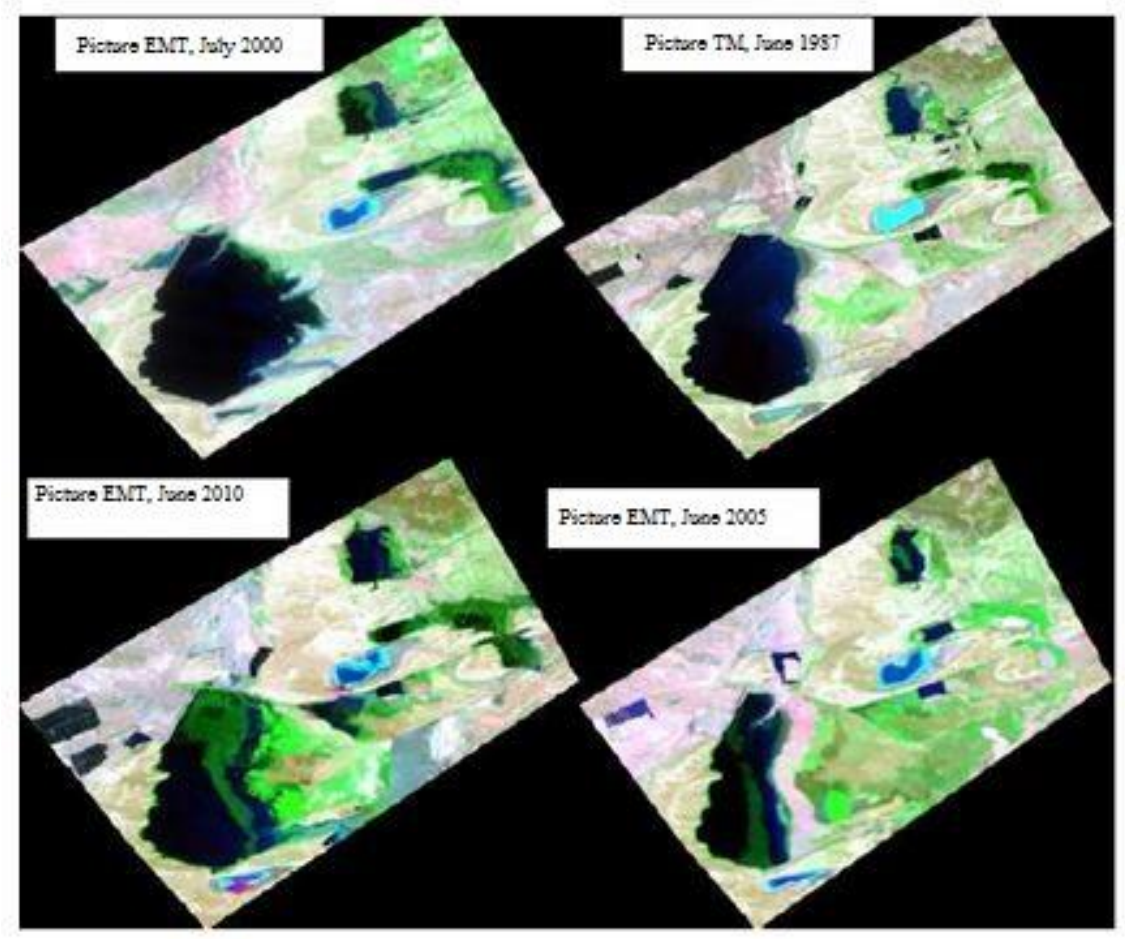

Figure 5: Visual changes of Alagol, Almagol and Ajigul international wetlands from 1987 to 2010.
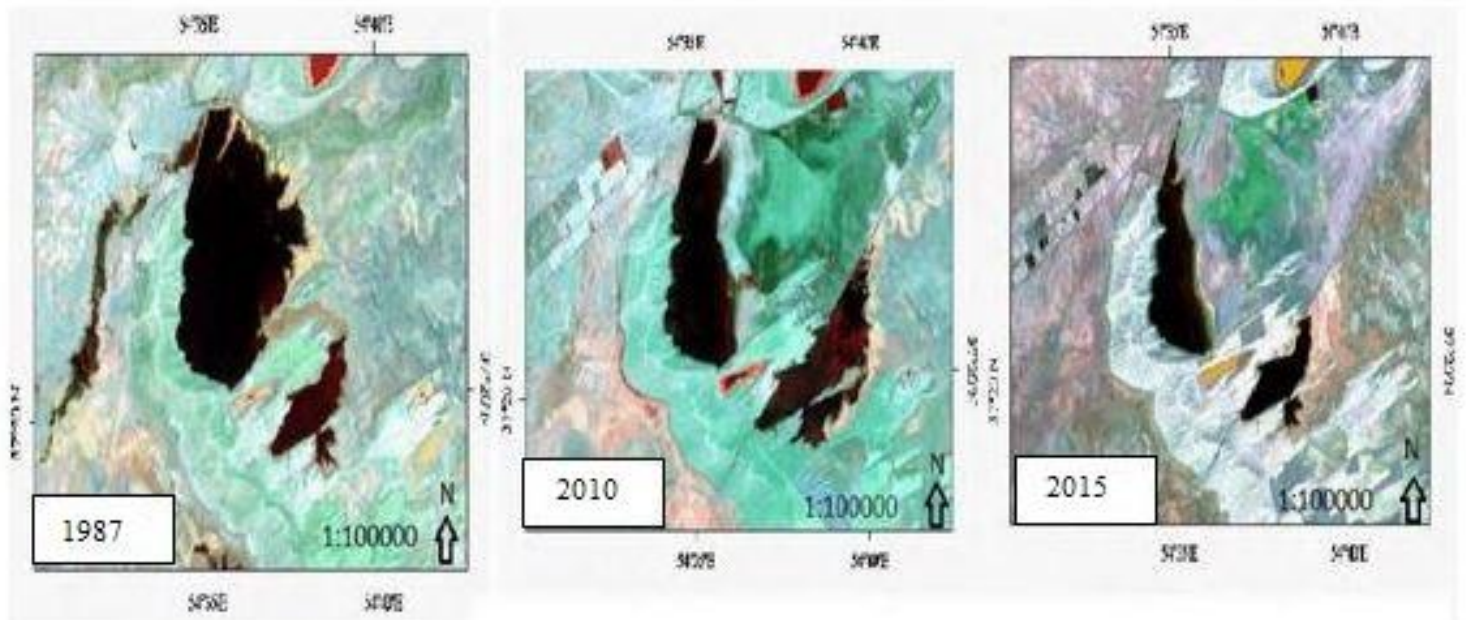

Figure 6: Trends of Alagol Wetland Changes from 1987 to 2015, Landsat satellite 


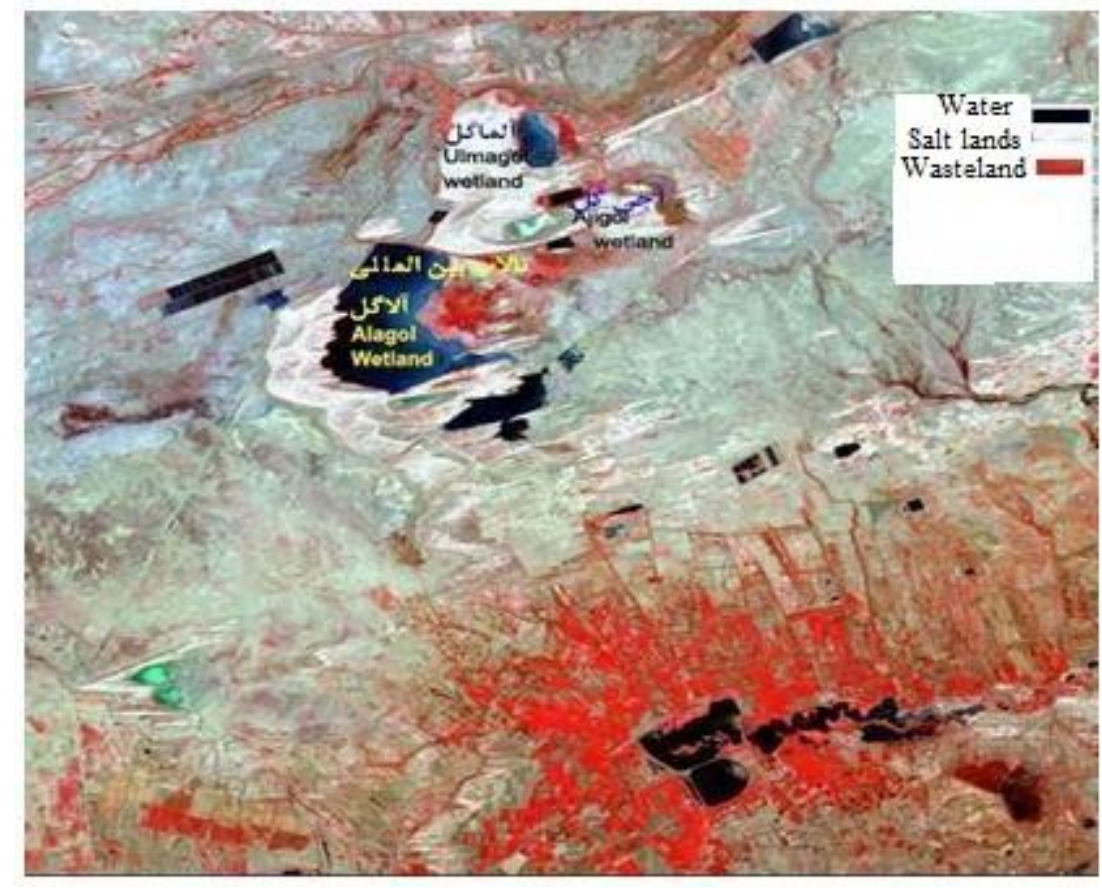

Figure 7: Virtual color combination RGB $=457$

By examining the maps and tables of changes, the following were obtained about the extent of changes in different uses:

The water class has declined $33.72 \%$ over the 13 years $(1987$ to 2000). Also, between 2005 and 2010, the water class dropped $48 \%$ in 2019 , it decreased to 906.3 hectare (10\%).

$\checkmark \quad$ Studies of the salt marshes of Almagol, Alagol, and Ajgul Wetlands showed that during 1987 to 2019, the salt class decreased from 523.4 hectares to 162 hectares in 2019.

High-density vegetation increased from 1247.6 hectares in 1987 to 2070.6 hectares in 2005 , but during
2005 to 2010 the vegetation class decreased significantly to 832.3 hectares in 2010 .

A study of the average vegetation showed that this cover was 1919.4 hectares in 1987, which decreased to 989.6 hectares in 2000 . Then increased to 3573 hectares until 2010. This has increased to 3691 in 2015 and 3708 hectares in 2019.

$$
\checkmark \text { Changes in vegetation }
$$
with low density have increased from an area of 976.2 hectares in 1987 to 1732.2 hectares in 2019.

$\checkmark \quad$ In this study, the area of wasteland increased from 5647.8 
Periódico do Núcleo de Estudos e Pesquisas sobre Gênero e Direito

Centro de Ciências Jurídicas - Universidade Federal da Paraíba

V. 9 - $\mathrm{N}^{\circ} 02$ - Ano 2020

ISSN | 2179-7137 | http://periodicos.ufpb.br/ojs2/index.php/ged/index

hectares in 1987 to 5821 hectares in

2019.

Table 2: Comparison of land use changes and vegetation of lands based on classified images,

The numbers are in hectares.

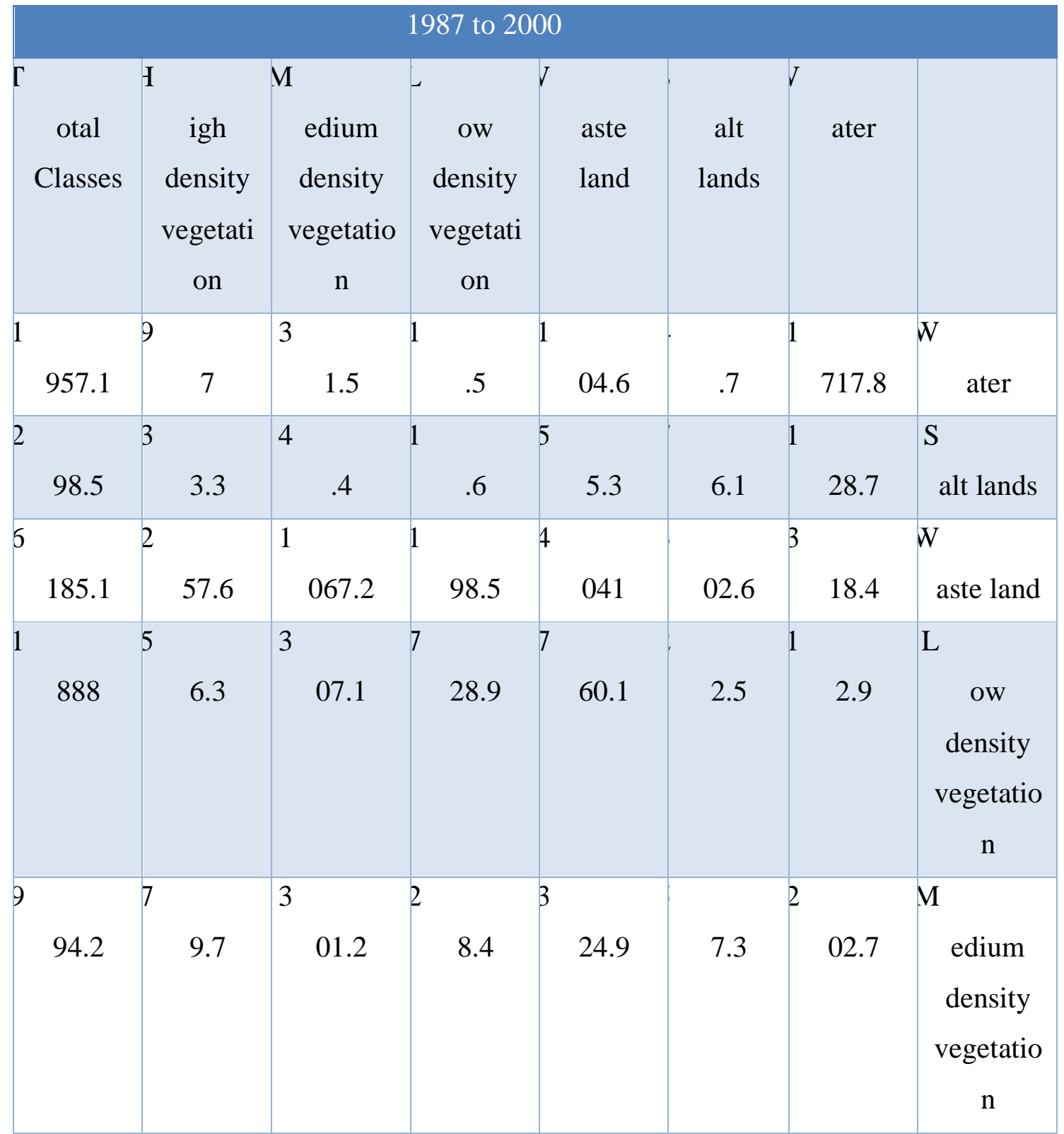


Periódico do Núcleo de Estudos e Pesquisas sobre Gênero e Direito

Centro de Ciências Jurídicas - Universidade Federal da Paraíba V. 9 - $\mathrm{N}^{\circ} 02$ - Ano 2020 ISSN | 2179-7137 | http://periodicos.ufpb.br/ojs2/index.php/ged/index

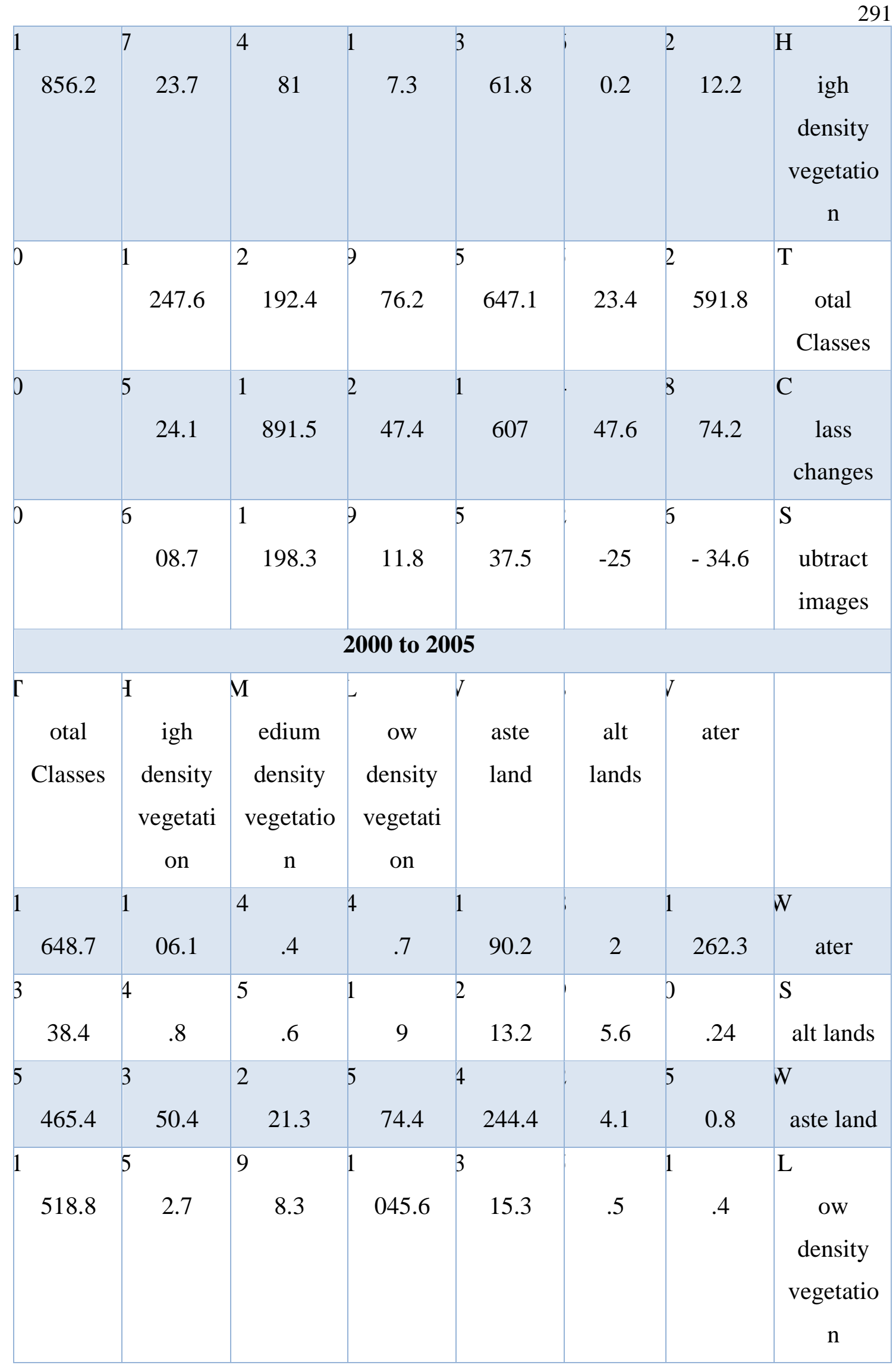


Periódico do Núcleo de Estudos e Pesquisas sobre Gênero e Direito

Centro de Ciências Jurídicas - Universidade Federal da Paraíba V. 9 - $\mathrm{N}^{\circ} 02$ - Ano 2020 ISSN | 2179-7137 | http://periodicos.ufpb.br/ojs2/index.php/ged/index

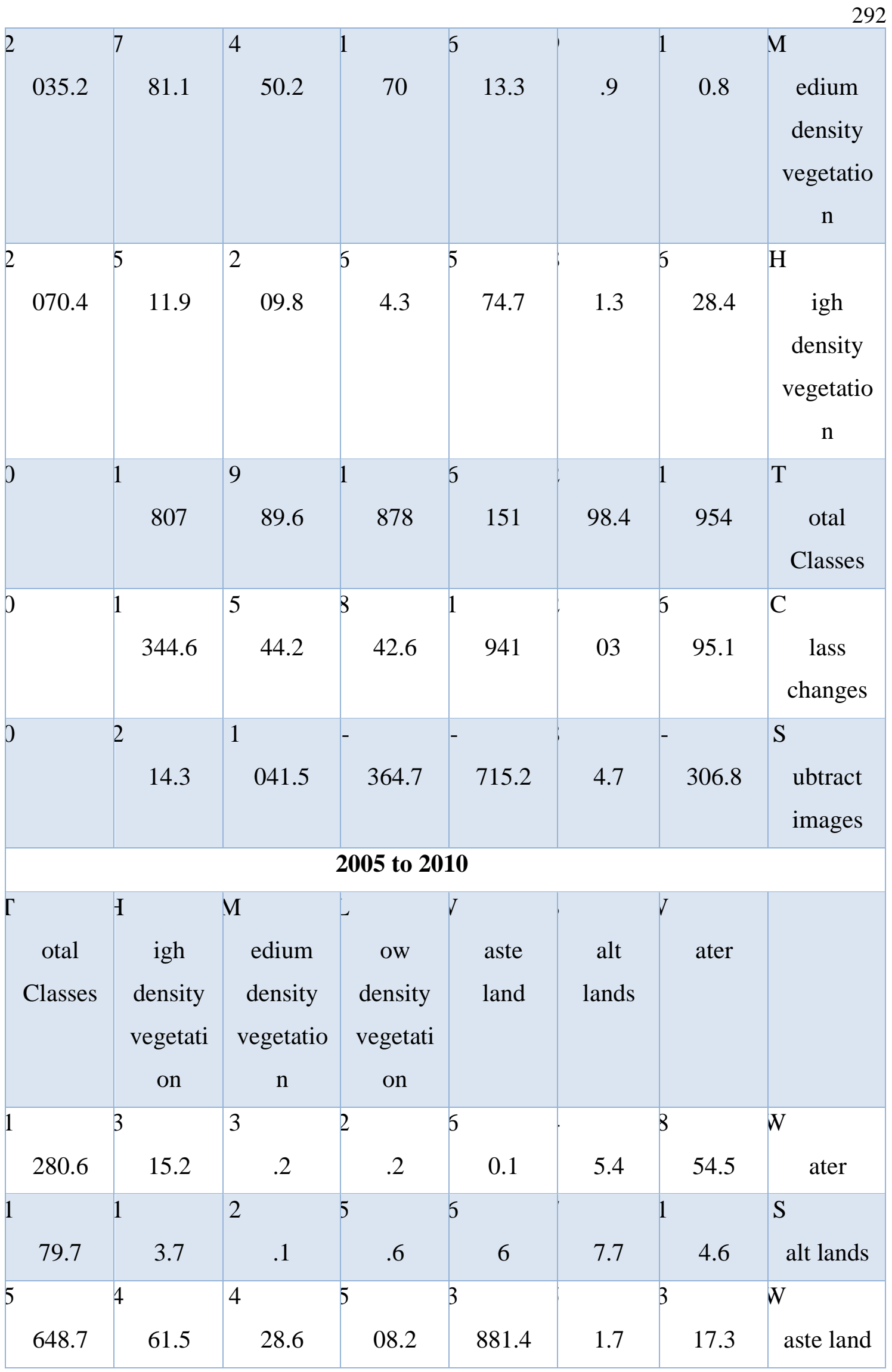


Periódico do Núcleo de Estudos e Pesquisas sobre Gênero e Direito Centro de Ciências Jurídicas - Universidade Federal da Paraíba V. 9 - $\mathrm{N}^{\circ} 02$ - Ano 2020 ISSN | 2179-7137 | http://periodicos.ufpb.br/ojs2/index.php/ged/index

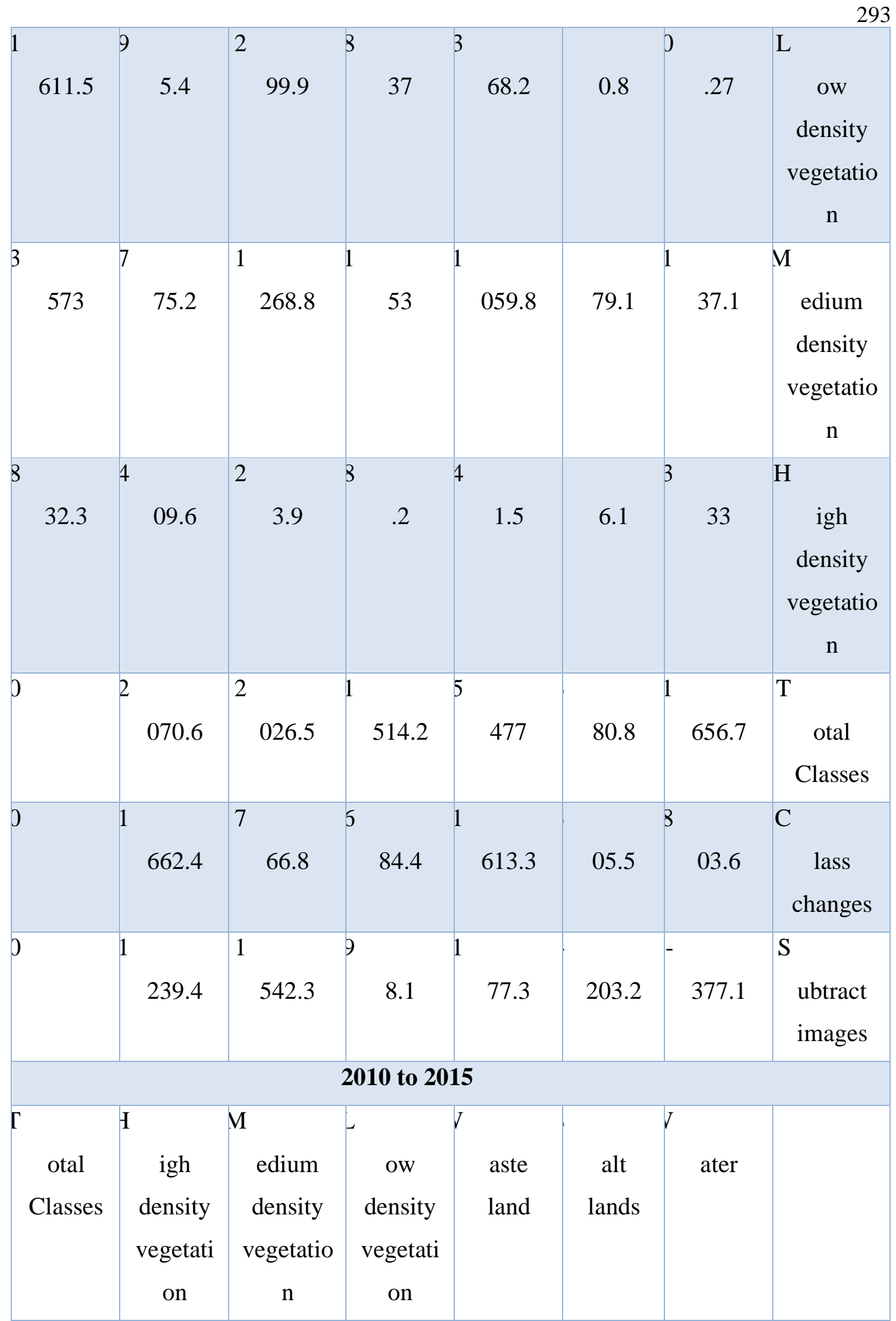


Periódico do Núcleo de Estudos e Pesquisas sobre Gênero e Direito

Centro de Ciências Jurídicas - Universidade Federal da Paraíba V. 9 - $\mathrm{N}^{\circ} 02$ - Ano 2020 ISSN | 2179-7137 | http://periodicos.ufpb.br/ojs2/index.php/ged/index

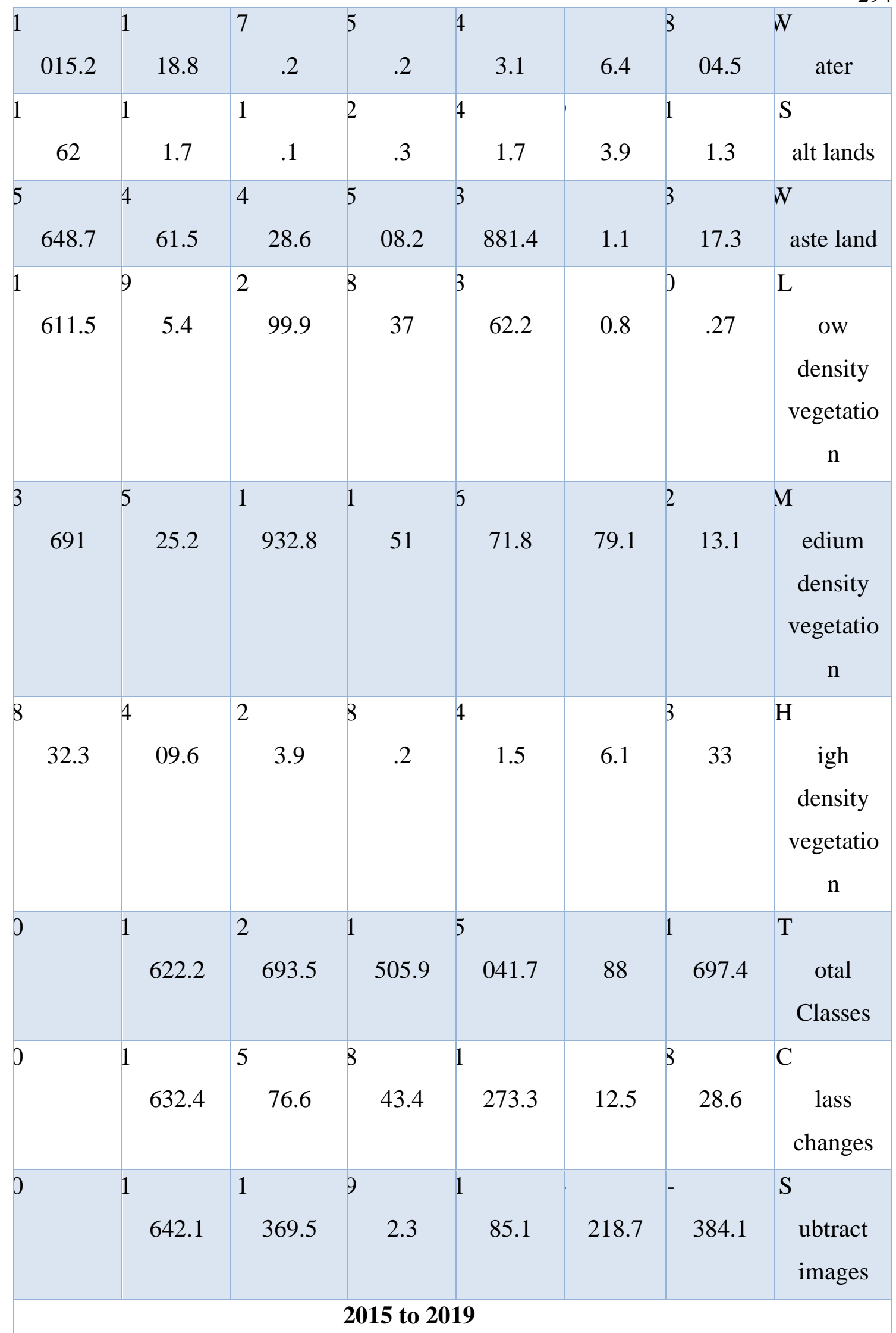


Periódico do Núcleo de Estudos e Pesquisas sobre Gênero e Direito

Centro de Ciências Jurídicas - Universidade Federal da Paraíba V. 9 - $\mathrm{N}^{\circ} 02$ - Ano 2020 ISSN | 2179-7137 | http://periodicos.ufpb.br/ojs2/index.php/ged/index

\begin{tabular}{|c|c|c|c|c|c|c|c|}
\hline$\Gamma$ & $\mathrm{I}$ & M & 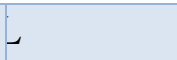 & $V$ & & & \\
\hline $\begin{array}{c}\text { otal } \\
\text { Classes }\end{array}$ & $\begin{array}{c}\text { igh } \\
\text { density } \\
\text { vegetati } \\
\text { on }\end{array}$ & $\begin{array}{c}\text { edium } \\
\text { density } \\
\text { vegetatio } \\
\text { n }\end{array}$ & $\begin{array}{c}\text { ow } \\
\text { density } \\
\text { vegetati } \\
\text { on }\end{array}$ & $\begin{array}{l}\text { aste } \\
\text { land }\end{array}$ & $\begin{array}{c}\text { alt } \\
\text { lands }\end{array}$ & ater & \\
\hline 9 & 9 & 1 & 3 & 4 & & & $W$ \\
\hline 06.3 & 0.9 & 2.2 & .6 & 8.1 & 9 & 12.5 & ater \\
\hline 1 & 1 & 1 & 1 & 4 & & & $\mathrm{~S}$ \\
\hline 62 & 6.3 & .8 & .6 & 9.1 & 4.9 & 8.3 & alt lands \\
\hline 5 & 8 & 5 & 4 & 3 & & & $W$ \\
\hline 821 & 83.5 & 18.6 & 89.2 & 578.6 & 2.3 & 98.8 & aste land \\
\hline 1 & 9 & 3 & 9 & 3 & & & $\mathrm{~L}$ \\
\hline 732.2 & 5.4 & 09.9 & 26 & 86.2 & 3.9 & .21 & $\begin{array}{c}\text { ow } \\
\text { density } \\
\text { vegetatio } \\
n\end{array}$ \\
\hline 3 & 9 & 1 & 1 & 7 & & & M \\
\hline 708 & 62.3 & 712.8 & 23 & 71.8 & 79.1 & 29.1 & $\begin{array}{c}\text { edium } \\
\text { density } \\
\text { vegetatio } \\
\mathrm{n}\end{array}$ \\
\hline 8 & 5 & 2 & 9 & 5 & & & $\mathrm{H}$ \\
\hline 32.3 & 41.9 & 6.4 & .3 & 3.7 & 2 & 79 & $\begin{array}{c}\text { igh } \\
\text { density } \\
\text { vegetatio } \\
\mathrm{n}\end{array}$ \\
\hline J & 2 & 2 & 1 & 3 & & & $\mathrm{~T}$ \\
\hline & 320.3 & 581.1 & 552.7 & 71.2 & 7.2 & 537.9 & $\begin{array}{c}\text { otal } \\
\text { Classes }\end{array}$ \\
\hline
\end{tabular}


Periódico do Núcleo de Estudos e Pesquisas sobre Gênero e Direito

Centro de Ciências Jurídicas - Universidade Federal da Paraíba

V. 9 - $\mathrm{N}^{\circ} 02$ - Ano 2020

ISSN | 2179-7137 | http://periodicos.ufpb.br/ojs2/index.php/ged/index

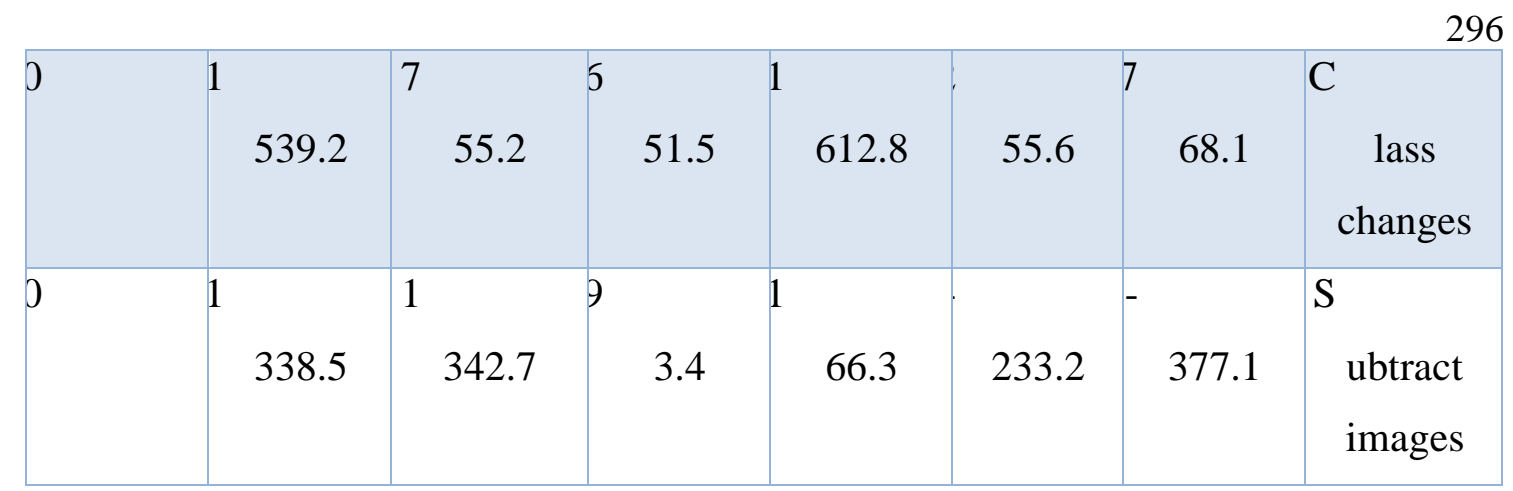

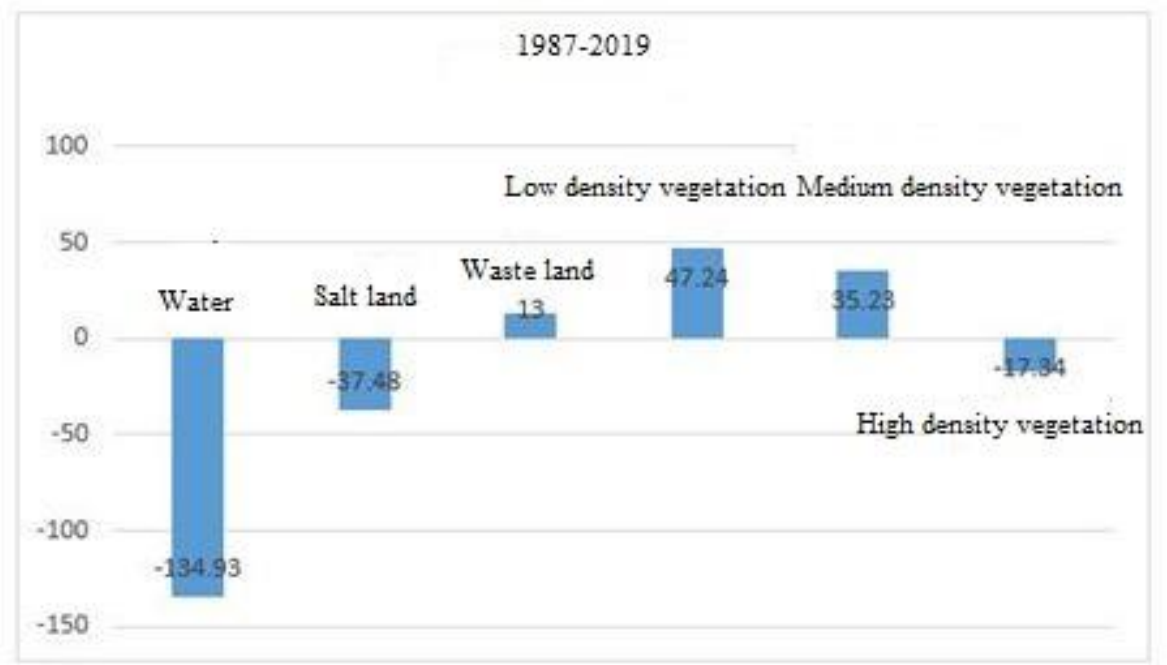

Chart 1: Changes in land use levels in Alagol, Ajigol and Almagol International Wetlands from 1987 to 2019 . Changes are expressed in percent. 


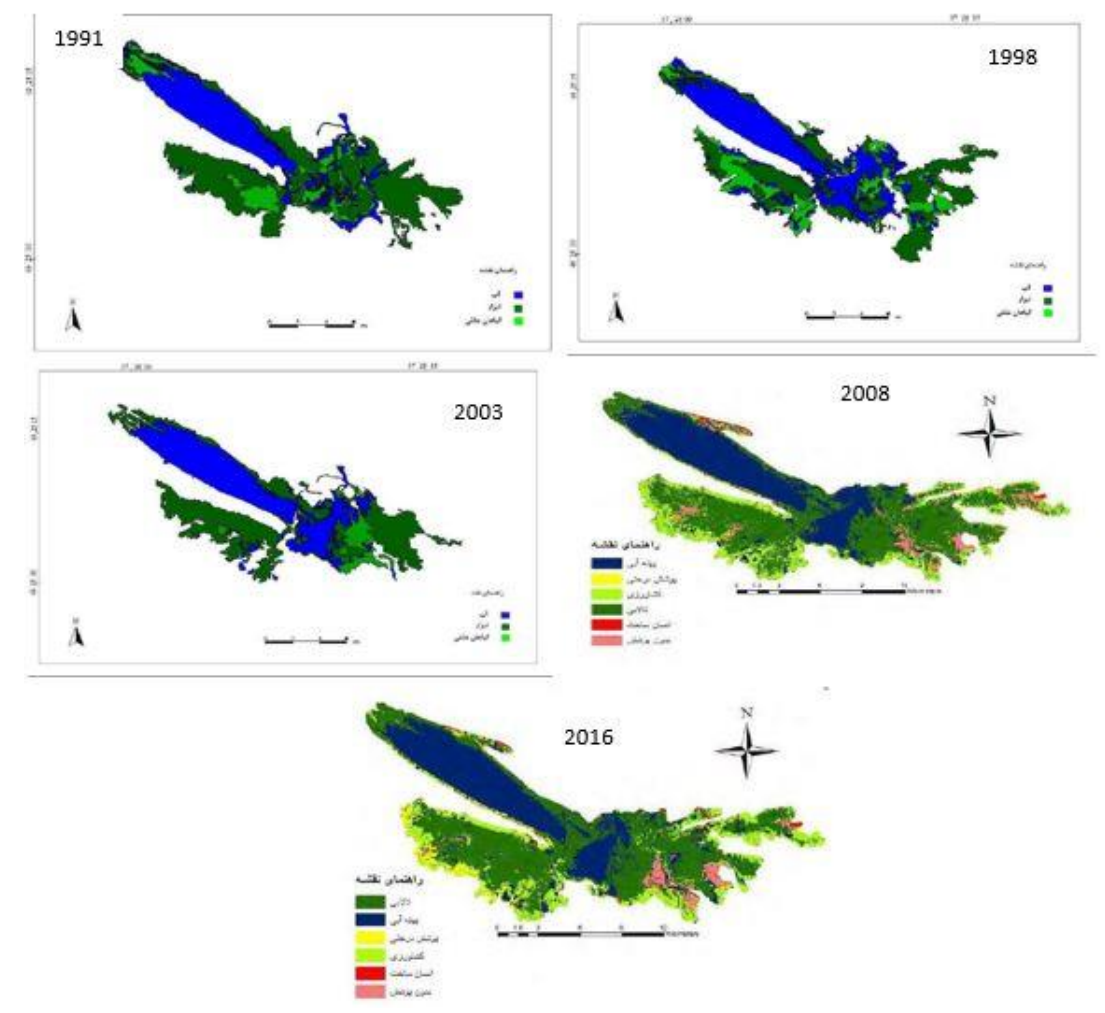

297

Figure 8: Anzali Wetland Range from 1991 to 2016 using Landsat satellite

The results of the interpretation of the images from Figures 1 to 5 were studied as RGB virtual color combinations (Fig. 9). In this picture, the water part and the herbaceous and hardwood plants are separated. 


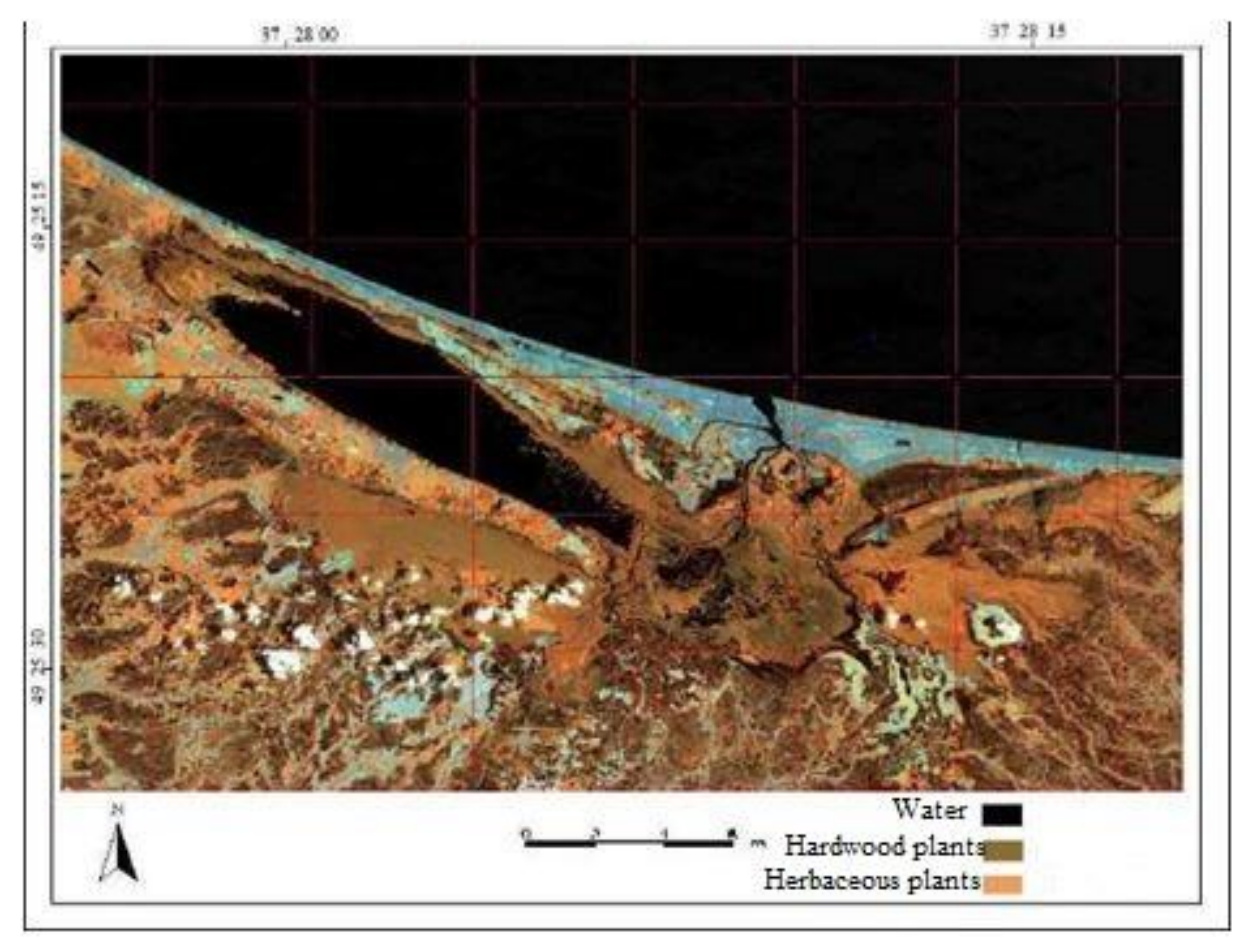

Figure 9: Virtual $\mathrm{RGB}=457$ color combination and vegetation resolution.

The results show that the area and width of the catchment area increased from 1991 to 1998 to $81 / 61$ square kilometers and then decreased until 2019. Also in the mud sector from 1991 to 2019 it has decreased from 17 square kilometers to 8.7 square kilometers. Over the years, the area of the pond and mud has declined, but the herbaceous section has increased from 40 square kilometers in 1998 to 148.1 square kilometers, in hardwood sector, it increased from 78 square kilometers to 139.6 square kilometers and in the human-made section from 1.7 square kilometers to 3.8 square kilometers.

Table 3: Comparison of land use changes and land vegetation cover based on Anzali wetland classified images using visual-numeral interpretation (numbers in square kilometers)

\begin{tabular}{|c|c|c|c|c|c|c|}
\hline \multicolumn{3}{|c|}{1991 to 1998} \\
\hline otal & H & H & He & van- \\
Classes & ardwood & rbaceous & ud & ater & \\
& & & & & \\
\hline
\end{tabular}


Periódico do Núcleo de Estudos e Pesquisas sobre Gênero e Direito

Centro de Ciências Jurídicas - Universidade Federal da Paraíba

V. 9 - $\mathrm{N}^{\circ} 02$ - Ano 2020

ISSN | 2179-7137 | http://periodicos.ufpb.br/ojs2/index.php/ged/index

\begin{tabular}{|c|c|c|c|c|c|c|}
\hline & & $\begin{array}{ll} & \\
& \\
\text { ants }\end{array}$ & $\begin{array}{l}\text { pla } \\
\\
\text { nts }\end{array}$ & & & \\
\hline $\begin{array}{ll}8 & \\
& 1.61\end{array}$ & $\begin{array}{ll}0 & \\
& \end{array}$ & $\begin{array}{ll}3 . & \\
& 1\end{array}$ & 6.6 & .7 & $\begin{array}{ll}6 & \\
& 3.3\end{array}$ & $\begin{array}{l}\mathrm{Wa} \\
\\
\text { ter }\end{array}$ \\
\hline 7 & $\begin{array}{ll}0 & \\
& .27\end{array}$ & $\begin{array}{l}0 . \\
\\
\end{array}$ & 1 & .3 & 6 & $\begin{array}{l}\mathrm{Mu} \\
\mathrm{d}\end{array}$ \\
\hline 0 & $\begin{array}{ll}1 & \\
& .1\end{array}$ & $\begin{array}{l}6 . \\
6\end{array}$ & $\begin{array}{r}12 . \\
8\end{array}$ & .3 & 2.9 & $\begin{array}{l}\text { He } \\
\text { rbaceous } \\
\text { pla } \\
\quad \text { nts }\end{array}$ \\
\hline 8 & 1 & $\begin{array}{ll}30 & \\
& .6\end{array}$ & $\begin{array}{l}24 . \\
2\end{array}$ & 3.3 & 9 & $\begin{array}{l}\text { Ha } \\
\text { rdwood } \\
\text { pla } \\
\quad \text { nts }\end{array}$ \\
\hline .7 & $\begin{array}{ll}0 & \\
& .6\end{array}$ & $\begin{array}{ll}0 . & \\
& \end{array}$ & 0.2 & .2 & .3 & $\begin{array}{l}\mathrm{Hu} \\
\text { man-made }\end{array}$ \\
\hline$\rho$ & $\begin{array}{ll}3 & \\
& .17\end{array}$ & $\begin{array}{ll}21 & \\
& .2\end{array}$ & $\begin{array}{r}44 . \\
8\end{array}$ & 9.8 & 2.4 & $\begin{array}{l}\text { Tot } \\
\quad \text { al } \\
\text { Classes }\end{array}$ \\
\hline$\rho$ & $\begin{array}{ll}1 & \\
& 3.6\end{array}$ & $\begin{array}{l}14 \\
.2\end{array}$ & $\begin{array}{l}28 . \\
9\end{array}$ & 4.3 & 8.2 & $\begin{array}{l}\text { Cla } \\
\text { ss changes }\end{array}$ \\
\hline p & $\begin{array}{ll}1 & \\
& 3.4\end{array}$ & $\begin{array}{ll}11 & \\
& .8\end{array}$ & $\begin{array}{l}18 . \\
1\end{array}$ & 21 & 23.6 & $\begin{array}{l}\mathrm{Su} \\
\text { btract } \\
\text { images }\end{array}$ \\
\hline \multicolumn{7}{|c|}{1998 to 2003} \\
\hline $\begin{array}{c}\text { otal } \\
\text { Classes }\end{array}$ & $\begin{array}{l}\mathrm{H} \\
\text { uman- } \\
\text { made }\end{array}$ & $\begin{array}{l}\text { ha } \\
\text { rdwood }\end{array}$ & $\begin{array}{l}\mathrm{He} \\
\text { rbaceous } \\
\text { pla } \\
\quad \text { nts }\end{array}$ & ud & ater & \\
\hline 6.10 & $\begin{array}{ll}0 & \\
& .4\end{array}$ & $\begin{array}{l}5 . \\
1\end{array}$ & 9.9 & .7 & 8.8 & $\begin{array}{l}\mathrm{Wa} \\
\\
\text { ter }\end{array}$ \\
\hline
\end{tabular}


Periódico do Núcleo de Estudos e Pesquisas sobre Gênero e Direito Centro de Ciências Jurídicas - Universidade Federal da Paraíba V. 9 - $\mathrm{N}^{\circ} 02$ - Ano 2020 ISSN | 2179-7137 | http://periodicos.ufpb.br/ojs2/index.php/ged/index

\begin{tabular}{|c|c|c|c|c|c|c|}
\hline 4 & \begin{tabular}{|lll}
0 & \\
& .27 \\
& & \\
&
\end{tabular} & $\begin{array}{ll}0 . & \\
& 7\end{array}$ & 1.2 & .6 & 3 & $\begin{array}{ll}\mathrm{Mu} & \\
\mathrm{d}\end{array}$ \\
\hline 11 & $\begin{array}{ll}1 & \\
& 0\end{array}$ & $\begin{array}{ll}11 & \\
& .9\end{array}$ & $\begin{array}{r}65 . \\
7\end{array}$ & 2.6 & 0.1 & $\begin{array}{l}\mathrm{He} \\
\text { rbaceous } \\
\text { pla } \\
\quad \text { nts }\end{array}$ \\
\hline 9 & 4 & $\begin{array}{ll}49 & \\
& .9\end{array}$ & $\begin{array}{ll}20 . & \\
& 2\end{array}$ & 6.4 & 7 & $\begin{array}{l}\text { har } \\
\text { dwood }\end{array}$ \\
\hline .1 & $\begin{array}{ll} & \\
& \\
& .2\end{array}$ & $\begin{array}{ll}0 . & \\
& 9\end{array}$ & 0.3 & .4 & .3 & $\begin{array}{l}\mathrm{Hu} \\
\text { man-made }\end{array}$ \\
\hline 0 & \begin{tabular}{|ll}
1 & \\
& 4.87
\end{tabular} & $\begin{array}{ll}68 & \\
& .5\end{array}$ & $\begin{array}{r}97 . \\
\\
\end{array}$ & 6.7 & 9.8 & $\begin{array}{l}\text { Tot } \\
\quad \text { al } \\
\text { Classes }\end{array}$ \\
\hline$\rho$ & $\begin{array}{ll}1 & \\
& 2.4\end{array}$ & $\begin{array}{ll}19 & \\
& .4\end{array}$ & $\begin{array}{l}20 . \\
\quad 3\end{array}$ & 2.5 & 2.3 & $\begin{array}{l}\text { Cla } \\
\text { ss changes }\end{array}$ \\
\hline 0 & $\begin{array}{|ll|}3 & \\
& 3.4 \\
\end{array}$ & $\begin{array}{ll}21 & \\
& \\
& 8\end{array}$ & $\begin{array}{l}16 . \\
6\end{array}$ & 19.4 & 13.2 & $\begin{array}{l}\mathrm{Su} \\
\text { btract } \\
\text { images }\end{array}$ \\
\hline \multicolumn{7}{|c|}{2003 to 2008} \\
\hline $\begin{array}{c}\text { otal } \\
\text { Classes }\end{array}$ & $\begin{array}{l}\text { H } \\
\text { uman- } \\
\text { made }\end{array}$ & $\begin{array}{l}\text { ha } \\
\text { rdwood }\end{array}$ & $\begin{array}{l}\mathrm{He} \\
\text { rbaceous } \\
\text { pla } \\
\quad \text { nts }\end{array}$ & ud & ater & \\
\hline 6.10 & $\begin{array}{lll}0 & & \\
& & \\
& .4\end{array}$ & $\begin{array}{ll}5 . & \\
& 1\end{array}$ & 9.9 & .7 & 8.8 & $\begin{array}{l}\mathrm{Wa} \\
\text { ter }\end{array}$ \\
\hline 0 & \begin{tabular}{|ll} 
& \\
& \\
& .34
\end{tabular} & $\begin{array}{l}1 . \\
\\
\end{array}$ & 0.2 & .6 & .3 & $\mathrm{Mu}_{\mathrm{d}}$ \\
\hline 23.8 & 1 & $\begin{array}{ll}8 . & \\
& 0\end{array}$ & $\begin{array}{l}89 . \\
\\
\end{array}$ & 5.3 & 8.4 & $\begin{array}{l}\mathrm{He} \\
\text { rbaceous } \\
\text { pla } \\
\quad \text { nts }\end{array}$ \\
\hline
\end{tabular}


Periódico do Núcleo de Estudos e Pesquisas sobre Gênero e Direito

Centro de Ciências Jurídicas - Universidade Federal da Paraíba

V. 9 - $\mathrm{N}^{\circ} 02$ - Ano 2020

ISSN | 2179-7137 | http://periodicos.ufpb.br/ojs2/index.php/ged/index

\begin{tabular}{|c|c|c|c|c|c|c|}
\hline 03.4 & 4 & $\begin{array}{ll}46 & \\
& .3\end{array}$ & $\begin{array}{l}29 . \\
\\
\\
\quad 2\end{array}$ & 6.4 & 7 & $\begin{array}{l}\text { har } \\
\text { dwood }\end{array}$ \\
\hline .9 & $\begin{array}{|ll|}1 & \\
& .3 \\
& \\
\end{array}$ & $\begin{array}{ll}0 . & \\
& 4\end{array}$ & 0.3 & .7 & .2 & $\begin{array}{l}\mathrm{Hu} \\
\text { man-made }\end{array}$ \\
\hline 0 & \begin{tabular}{|ll}
7 & \\
& \\
& .04
\end{tabular} & $\begin{array}{ll}\begin{array}{ll}61 \\
\end{array} & \\
& .5\end{array}$ & $\begin{array}{ll}12 & \\
& 8.8\end{array}$ & 5.7 & 6 & $\begin{array}{l}\text { Tot } \\
\text { al } \\
\text { Classes }\end{array}$ \\
\hline 0 & $\begin{array}{ll}3 & \\
& 2.9\end{array}$ & $\begin{array}{ll}18 & \\
& .2\end{array}$ & $\begin{array}{l}27 . \\
\\
\end{array}$ & 9.6 & 8.1 & $\begin{array}{l}\text { Cla } \\
\text { ss changes }\end{array}$ \\
\hline 0 & $\begin{array}{|ll|}2 & \\
& 3.4 \\
& \\
\end{array}$ & $\begin{array}{ll}11 & \\
& .8\end{array}$ & $\begin{array}{l}12 . \\
6\end{array}$ & 13.2 & 23.8 & $\begin{array}{l}\mathrm{Su} \\
\text { btract } \\
\text { images }\end{array}$ \\
\hline \multicolumn{7}{|c|}{2008 to 2019} \\
\hline $\begin{array}{c}\text { otal } \\
\text { Classes }\end{array}$ & $\begin{aligned} & \text { H } \\
& \text { uman- } \\
& \text { made }\end{aligned}$ & $\begin{array}{l}\text { ha } \\
\text { rdwood }\end{array}$ & $\begin{array}{l}\mathrm{He} \\
\text { rbaceous } \\
\text { pla } \\
\quad \text { nts }\end{array}$ & ud & ater & \\
\hline 6.2 & $\begin{array}{ll}1 & \\
& 6.4\end{array}$ & $\begin{array}{ll}1 . & \\
& 1\end{array}$ & 3.9 & .7 & 4.8 & $\begin{array}{l}\mathrm{Wa} \\
\text { ter }\end{array}$ \\
\hline .7 & $\begin{array}{ll}7 & \\
& .0\end{array}$ & $\begin{array}{ll}1 . & \\
& 3\end{array}$ & 0.5 & .9 & .3 & $\mathrm{Mu}_{\mathrm{d}}$ \\
\hline 48.1 & $\begin{array}{ll}1 & \\
& 3\end{array}$ & $\begin{array}{l}4 . \quad \\
4\end{array}$ & $\begin{array}{r}96 . \\
\\
\end{array}$ & 8.4 & 1 & $\begin{array}{l}\mathrm{He} \\
\text { rbaceous } \\
\text { pla } \\
\quad \text { nts }\end{array}$ \\
\hline 39.6 & $\begin{array}{ll}1 & \\
& 4.1\end{array}$ & $\begin{array}{ll}79 & \\
& .7\end{array}$ & $\begin{array}{l}37 . \\
1\end{array}$ & 9.2 & .3 & $\begin{array}{l}\text { har } \\
\text { dwood }\end{array}$ \\
\hline .8 & $\begin{array}{lll}1 & & \\
& .9\end{array}$ & $\begin{array}{ll}0 . & \\
& 9\end{array}$ & 0.2 & .6 & .21 & $\begin{array}{l}\mathrm{Hu} \\
\text { man-made }\end{array}$ \\
\hline
\end{tabular}


Periódico do Núcleo de Estudos e Pesquisas sobre Gênero e Direito

Centro de Ciências Jurídicas - Universidade Federal da Paraíba

V. 9 - $\mathrm{N}^{\circ} 02$ - Ano 2020

ISSN | 2179-7137 | http://periodicos.ufpb.br/ojs2/index.php/ged/index

\begin{tabular}{|c|c|c|c|c|c|c|}
\hline 0 & \begin{tabular}{|ll}
5 & \\
& 2.4
\end{tabular} & $\begin{array}{ll}87 & \\
& .4\end{array}$ & $\begin{array}{ll}13 & \\
& 8\end{array}$ & 2.8 & 5.6 & $\begin{array}{l}\text { Tot } \\
\text { al } \\
\text { Classes }\end{array}$ \\
\hline$\rho$ & $\begin{array}{|ll|}3 & \\
& 2.9\end{array}$ & $\begin{array}{ll}28 & \\
& .2\end{array}$ & $\begin{array}{l}37 . \\
3\end{array}$ & 5.3 & 8.7 & $\begin{array}{l}\text { Cla } \\
\text { ss changes }\end{array}$ \\
\hline 0 & $\begin{array}{|ll|}2 & \\
& \\
& 4.1 \\
\end{array}$ & $\begin{array}{ll}22 & \\
& .8\end{array}$ & $\begin{array}{l}16 . \\
6\end{array}$ & 23.2 & 13.8 & $\begin{array}{l}\mathrm{Su} \\
\text { btract } \\
\text { images }\end{array}$ \\
\hline
\end{tabular}

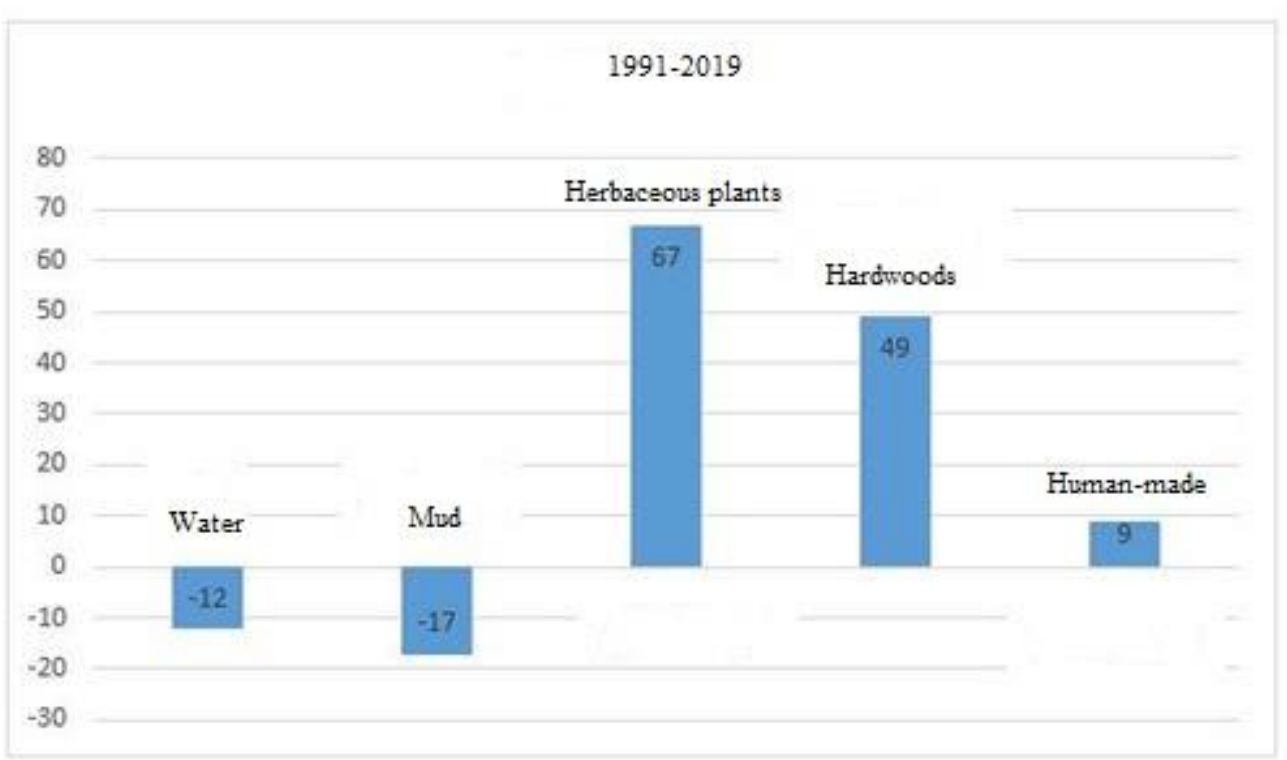

Chart 2: Changes in land use levels in the Anzali Wetland area from 1991 to 2019. Changes are expressed as percentage.

Table 4: Estimation of relative vegetation cover percentage of transects in

Almagol, Ajgul and Alagol wetlands

\begin{tabular}{|l|c|c|c|}
\hline $\begin{array}{c}\text { Relative } \\
\text { percentage }\end{array}$ & $\begin{array}{c}\text { Coverage } \\
\text { percent }\end{array}$ & $\begin{array}{c}\text { Wetland } \\
\text { index }\end{array}$ & Name \\
\hline 61 & 63 & $\begin{array}{c}\text { Non- } \\
\text { wetland }\end{array}$ & Pruritus \\
\hline
\end{tabular}


Periódico do Núcleo de Estudos e Pesquisas sobre Gênero e Direito

Centro de Ciências Jurídicas - Universidade Federal da Paraíba

V. 9 - $\mathrm{N}^{\circ} 02$ - Ano 2020

ISSN | 2179-7137 | http://periodicos.ufpb.br/ojs2/index.php/ged/index

\begin{tabular}{|c|c|c|c|}
\hline 19.9 & 20 & Wetland & $\begin{array}{l}\text { Atriplex } \\
\text { lentiformis }\end{array}$ \\
\hline 2.9 & 3 & $\begin{array}{l}\text { Non- } \\
\text { wetland }\end{array}$ & $\begin{array}{l}\text { Salsola } \\
\text { Rigida }\end{array}$ \\
\hline 10.2 & 10.5 & Wetland & French Tama \\
\hline 13.5 & 38 & Wetland & $\begin{array}{l}\text { Tamarix } \\
\text { ramosissima }\end{array}$ \\
\hline 13.5 & 38 & Wetland & $\begin{array}{l}\text { Limonium } \\
\text { reniformis }\end{array}$ \\
\hline 24 & 63 & $\begin{array}{l}\text { Non- } \\
\text { wetland }\end{array}$ & $\begin{array}{l}\text { Halostachys } \\
\text { belangeriana }\end{array}$ \\
\hline 0.8 & 85.5 & $\begin{array}{l}\text { Non- } \\
\text { wetland }\end{array}$ & $\begin{array}{l}\text { Helxine } \\
\text { Soleirolii \& Lichen }\end{array}$ \\
\hline 3.5 & 3 & $\begin{array}{l}\text { Non- } \\
\text { wetland }\end{array}$ & Geraniacea \\
\hline 0 & 85 & $\begin{array}{l}\text { Non- } \\
\text { wetland }\end{array}$ & Atriplex \\
\hline
\end{tabular}

Table 5: Estimation of relative vegetation cover percentage in transects in

Anzali Wetland

\begin{tabular}{|c|c|c|c|}
\hline $\begin{array}{c}\text { Relative } \\
\text { percentage }\end{array}$ & $\begin{array}{c}\text { Coverage } \\
\text { percentage }\end{array}$ & $\begin{array}{c}\text { Wetland } \\
\text { index }\end{array}$ & Name \\
\hline 41 & 47 & Wetland & $\begin{array}{r}\text { Phragmites } \\
\text { australis }\end{array}$ \\
\hline 19.9 & 19 & Wetland & Sparaganium \\
\hline 5.9 & 6 & Non- & $\begin{array}{c}\text { Typha } \\
\text { latifolia }\end{array}$ \\
\hline 10.2 & 10.5 & wetland & $\begin{array}{c}\text { Scirpus } \\
\text { cernuus }\end{array}$ \\
\hline
\end{tabular}


Periódico do Núcleo de Estudos e Pesquisas sobre Gênero e Direito

Centro de Ciências Jurídicas - Universidade Federal da Paraíba

V. 9 - $\mathrm{N}^{\circ} 02$ - Ano 2020

ISSN | 2179-7137 | http://periodicos.ufpb.br/ojs2/index.php/ged/index

\begin{tabular}{|c|c|c|c|}
\hline 23.5 & 27 & Wetland & $\begin{array}{c}\text { Polygonum } \\
\text { Capitatum }\end{array}$ \\
\hline 21.1 & 27 & Wetland & $\begin{array}{c}\text { Mentha } \\
\text { aquatica }\end{array}$ \\
\hline 2.1 & 4 & Wetland & $\begin{array}{c}\text { Sagittaria } \\
\text { trifolia }\end{array}$ \\
\hline 46 & 54 & Wetland & $\begin{array}{c}\text { Potamogeton } \\
\text { crispus }\end{array}$ \\
\hline 28.8 & 36.5 & Wetland & Butomus \\
& & & umbellatus \\
\hline 3.5 & 3 & Wetland & nelumbo \\
\hline 12 & 12.12 & Wetland & Utricularia \\
\hline 11 & 13.7 & Wetland & Shalovin \\
\hline 11.5 & 28 & Wetland & hydrocharis \\
\hline 21.9 & 56 & Wetland & Salvia \\
\hline 47.7 & & & Field horsetail \\
\hline & & & \\
\hline
\end{tabular}

Table 6: List of the genus \& species of wintering water birds \& waders in

Almagol wetland, Ajigul and Alagol 2019.

\begin{tabular}{|c|c|c|c|c|c|c|}
\hline $\begin{array}{l}\mathrm{Sa} \\
\text { ndpiper }\end{array}$ & arus & uck & $\begin{array}{l}\text { Co } \\
\text { rmorant }\end{array}$ & $\begin{array}{l}\mathrm{P} \\
\text { elican }\end{array}$ & $\begin{array}{l}\mathrm{P} \\
\text { odiceps } \\
\text { nigricollis }\end{array}$ & \\
\hline 1 & $\beta$ & 1 & 1 & 0 & 0 & $\begin{array}{l}\text { A } \\
\quad \text { lagol }\end{array}$ \\
\hline 3 & 2 & 5 & 0 & 0 & 0 & $\begin{array}{l}\text { A } \\
\text { lmagol }\end{array}$ \\
\hline 2 & 2 & $p$ & 1 & 2 & 4 & A \\
\hline
\end{tabular}


Table 7: List of the genus \& species of wintering water birds \& waders in

Anzali Wetland 2019

\begin{tabular}{|c|c|c|}
\hline Status & Species & Genus \\
\hline 5 & pelican white & \multirow[b]{2}{*}{ Pelecanidae } \\
\hline 1123 & Dalmatian pelican & \\
\hline 953 & $\begin{array}{r}\text { phalacrocorax } \\
\text { pygmeus }\end{array}$ & \multirow[t]{2}{*}{ Phalacrocoracidae } \\
\hline 3642 & $\begin{array}{r}\text { Phalacrocorax } \\
\text { carbo }\end{array}$ & \\
\hline 23 & Ardea cinerea & Ardeidae \\
\hline 2300 & Anser anser & \multirow{10}{*}{ Podicipediformes } \\
\hline 96 & Cygnus cygnus & \\
\hline 3 & Tadorna tadorna & \\
\hline 2472 & Gadwall & \\
\hline 2533 & Anas crecca & \\
\hline 7591 & Anas platyrhynchos & \\
\hline 13 & Northern shoveler & \\
\hline 481 & Aythya ferina & \\
\hline 11 & Aythya nyroca & \\
\hline 162 & Tufted duck & \\
\hline 1592 & Vanellus vanellus & Charadriidae \\
\hline 55 & Tringa totanus & \multirow[b]{2}{*}{ Scolopacidae } \\
\hline 74 & Gallinago gallinago & \\
\hline 37 & $\begin{array}{l}\text { Chlidonias } \\
\text { hybridus }\end{array}$ & strenidae \\
\hline 23166 & - & Total \\
\hline
\end{tabular}




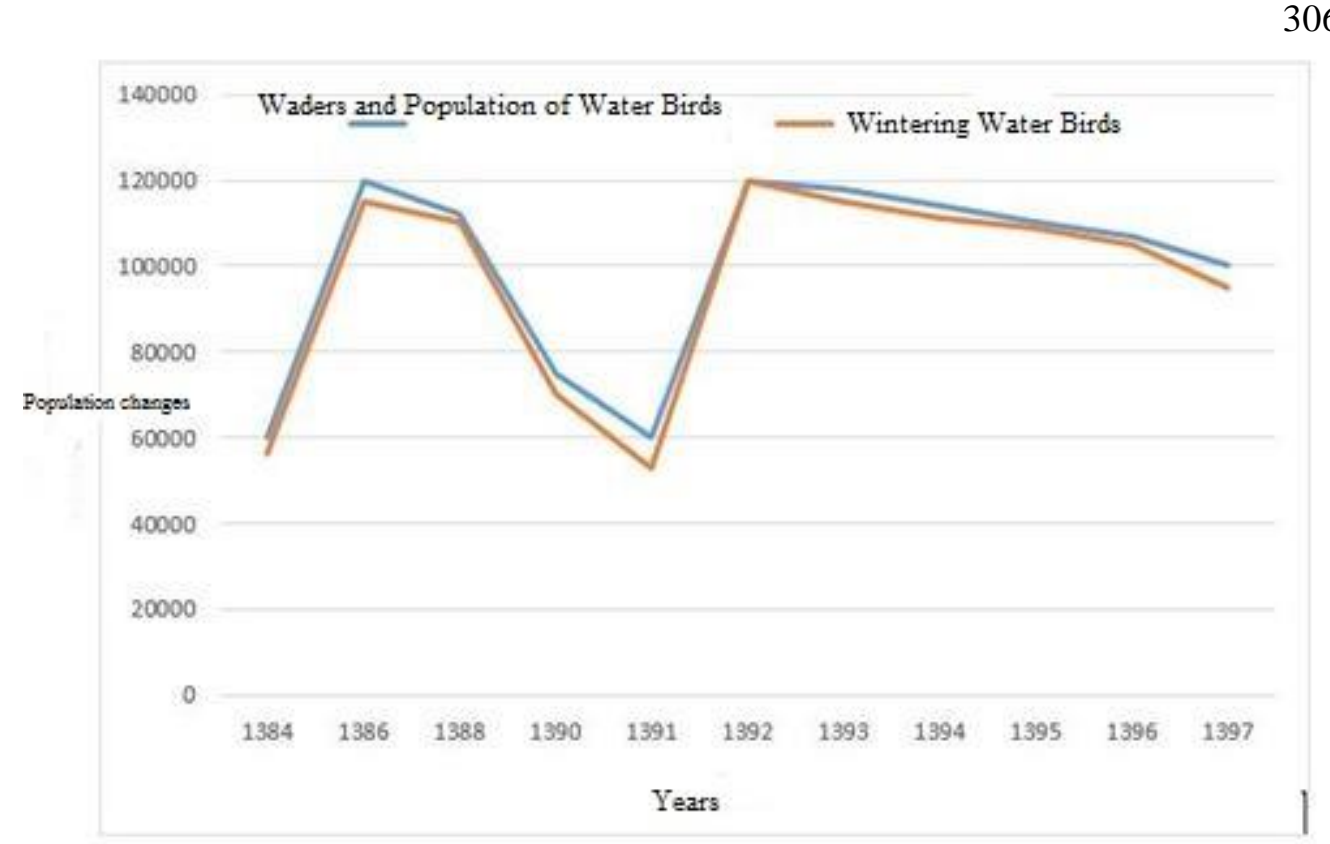

Chart 3: Population changes of wintering water birds \& waders and population of water birds Anzali Wetland, Almagol, Alagol and Ajigol

Table 8: Species richness index, species diversity and species uniformity of migratory and aquatic migratory birds overwintering to Anzali, Almagol, Alagol and Ajgol wetlands.

\begin{tabular}{|c|c|c|c|c|c|c|c|}
\hline $\begin{array}{c}\text { he } \\
\text { correl } \\
\text { ation } \\
\text { coeffi } \\
\text { cient }\end{array}$ & $\begin{array}{c}\text { ercent } \\
\text { of } \\
\text { varianc } \\
\text { e }\end{array}$ & $\begin{array}{l}\text { imons } \\
\text { unifor } \\
\text { mity }\end{array}$ & $\begin{array}{l}\text { U } \\
\text { niformity } \\
\text { of pilo }\end{array}$ & $\begin{array}{l}\text { imson } \\
\text { Index }\end{array}$ & $\begin{array}{l}\text { S } \\
\text { hannon- } \\
\text { Wiener }\end{array}$ & argalph & $\begin{array}{l}\text { Gen } \\
\text { us }\end{array}$ \\
\hline .7 & 6.5 & .52 & $\begin{array}{ll} & \\
& .67\end{array}$ & .6 & .08 & .12 & $\begin{array}{l}\text { Pel } \\
\text { ecanidae }\end{array}$ \\
\hline .74 & 2.4 & .87 & $\begin{array}{ll} & \\
& .89\end{array}$ & .75 & .62 & .09 & $\begin{array}{l}\text { Pha } \\
\text { lacrocoracid } \\
\text { ae }\end{array}$ \\
\hline
\end{tabular}


Periódico do Núcleo de Estudos e Pesquisas sobre Gênero e Direito

Centro de Ciências Jurídicas - Universidade Federal da Paraíba V. 9 - $\mathrm{N}^{\circ} 02$ - Ano 2020

ISSN | 2179-7137 | http://periodicos.ufpb.br/ojs2/index.php/ged/index

\begin{tabular}{|c|c|c|c|c|c|c|c|}
\hline .46 & 6.7 & .26 & \begin{tabular}{|ll}
0 & \\
& .48
\end{tabular} & .8 & .94 & 0 & $\begin{array}{l}\text { Ard } \\
\text { eidae }\end{array}$ \\
\hline .39 & 6.7 & .15 & \begin{tabular}{|ll}
0 & \\
& .45
\end{tabular} & .8 & .35 & .36 & $\begin{array}{l}\text { Pod } \\
\text { icipediforme } \\
\quad \mathrm{s}\end{array}$ \\
\hline .86 & 5.7 & .16 & \begin{tabular}{|ll} 
O & \\
& .39 \\
& .39
\end{tabular} & .4 & .15 & .57 & $\begin{array}{l}\text { Sco } \\
\text { lopacidae }\end{array}$ \\
\hline .88 & 3.2 & .38 & \begin{tabular}{|ll}
$\mathbf{0}$ & \\
& \\
& .57
\end{tabular} & .7 & .11 & .59 & lari \\
\hline .67 & 4.2 & .24 & \begin{tabular}{|ll}
0 & \\
& .46 \\
&
\end{tabular} & .3 & 1 & .35 & $\begin{array}{l}\text { stre } \\
\text { nidae }\end{array}$ \\
\hline .98 & 5.5 & .2 & \begin{tabular}{|ll}
$\mathbf{0}$ & \\
& $\mathbf{. 0 3}$
\end{tabular} & .09 & .06 & .39 & $\begin{array}{l}\text { Cha } \\
\text { radriidae }\end{array}$ \\
\hline .67 & 5.6 & .52 & \begin{tabular}{|ll}
0 & \\
& .67
\end{tabular} & .6 & .08 & .61 & $\begin{array}{l}\text { Pod } \\
\text { iceps } \\
\text { nigricollis }\end{array}$ \\
\hline
\end{tabular}

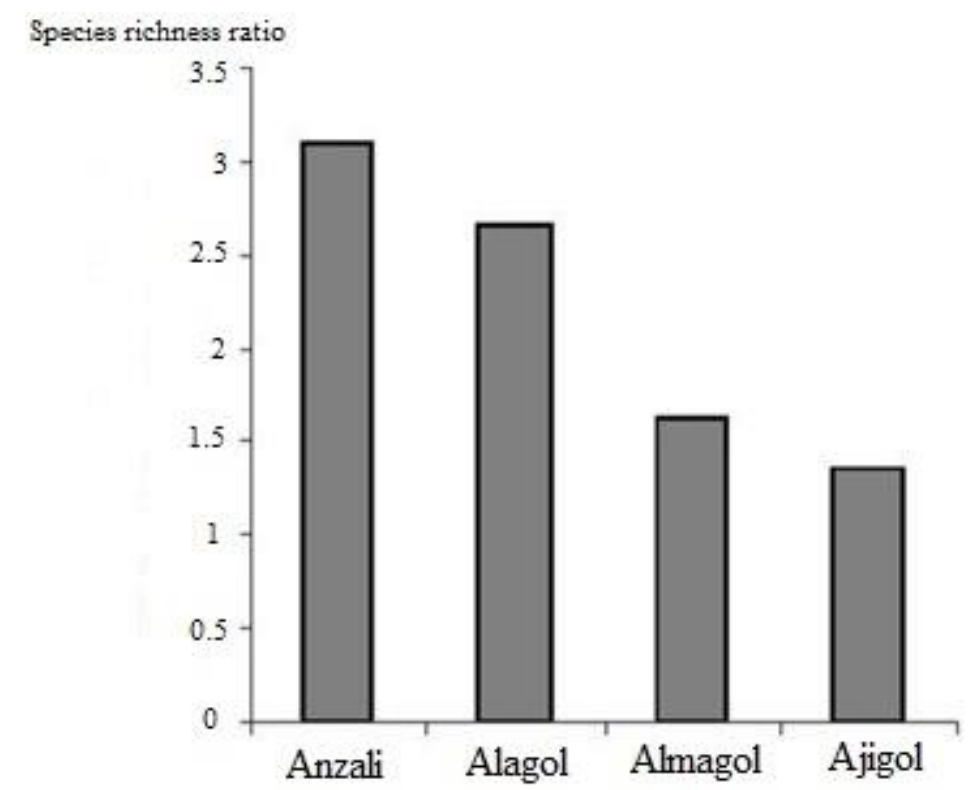

Chart 4: Species richness ratio of water birds to waders in the studied wetlands. 


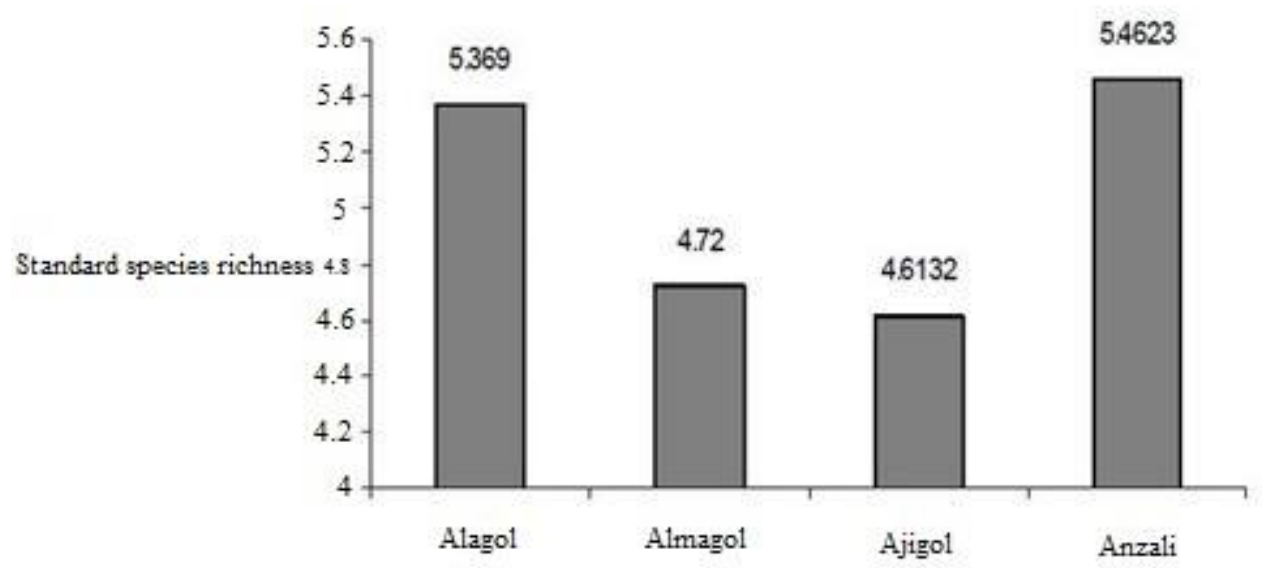

Chart 5: Standard species richness in the studied wetlands.

According to the Camargo

species uniformity index, the highest uniformity species of water birds and waders in the studied wetlands belonged to Ajigol wetland (0.206) and according to the Simpson Species Uniformity Index, the highest amount of species uniformity was in the same wetland with 0.109 .

\section{Discussion}

Activities such as incorrect water supply policies, barrier projects, road development, directing urban and industrial wastewater and industrial waste to wetlands and introducing nonnative species have put nearly all of Iran's international wetlands on the brink of extinction (Hosseini Mousavi, 2012). In the last decade, climate change, rainfall, human activities and mismanagement have reduced the water inside the wetlands and have led to seasonal or permanent drying of the wetlands. Understanding the process of change and understanding the evolution of ecosystems in general, and wetlands in particular, can be partially predictive of their future status if the current trend continues (Hosseini Mousavi, 2012). The use of remote sensing techniques is one of the most important and precise tools for performing these monitoring (Hosseini Mousavi, 2012). The results of the interpretation of satellite images in the years under study indicate that the area of the Anzali wetland was the highest in 1998 and the lowest in 1991. This is due to the outbreak of Caspian Sea water in 1998 and the droughts of the 
time period close to 1991 . On the other hand, all three wetlands of Alagol, Almagol and Ajigal have undergone extensive changes and their area has decreased from 2591.8 hectares to 906 hectares. Investigating the causes of these changes can be classified into two general categories of natural and human factors, such as: drought, dam construction, fish farming, irregular water harvesting for agriculture and canalization, and road construction (Hossein Jani, Ahmad Nejad and Mehdi Zadeh, 2016).

In Almagol, Alagol, and Ajlgol wetlands, vegetation distribution is narrower and changes in plant species occur on gradients from wetland to drought, at intervals of less than $30 \mathrm{~m}$. The results of this study emphasize that Landsat images along with ground sampling provide relatively accurate maps which have been confirmed in other researches (Hossein Jani, Ahmad Nejad and Mehdi Zadeh, 2016). According to the results of this study, the vegetation margins of the adjacent wetlands of the adjacent are very limited, but in some parts especially in the east of the wetland, Tamarisk and reed are very scattered in which non-growing seasons are predominant. The results also indicate that due to the lack of legal compliance with wetlands and increasing agricultural and man-made land uses, the effects of wetland ecosystems have been neglected in recent years due to factors such as the entry of different types of wastewater, the conversion of parts of the wetland. They are exposed to arable land, sedimentation of incoming water sediments, thereby reducing their surface area as well as the indiscriminate growth of aquatic and non-native plants.

Birds are one of the most important indicators of wetland habitat suitability. Therefore, the study of the changes in the diversity and population fluctuations of aquatic birds in different years can be considered as a biological indicator of the health status or the presence of threats in wetland ecosystems (Riazi and Mirmandehi, 2008). Riazi and Mirmandehi (2008), analyzing Mid-Winter census statistics of migratory water birds in 2000-2005 in wetlands of three northern provinces of Iran, stated that Anzali wetland along with Gomishan wetland had the most ecological value among wetlands in 
northern iran migratory aquaculture has overwintering.

Also Behroozirad (1999), during a study in a part of salekeh and esfand wetlands, they identified and categorized 59 species of aquatic and wader birds with monthly mean abundance of 5021 and 39 species and mean abundance of 2695 , respectively. In 2007 to 2015 , a total of 70 aquatic and wader bird species, including 37 aquatic and 33 wader species, were identified and censored. These birds belonged to 12 genera and ducks with 19 species had the highest number of species. The number of species of migratory aquatic and migratory waders has fluctuated during these years. The population of winter migratory aquatic birds has also fluctuated over the years in the Anzali Wetland, with the highest population of aquatic birds in winter 2015 and the lowest number in winter 2005 (101894) (Hossein Jani, Ahmad Nejad and Mehdi Zadeh, 2016). Aquatic birds had the highest number of migratory birds enumerated in Anzali wetland in all years compared to waders. In other words, an average of $94.75 \%$ of the winter migratory birds population belonged to aquatic birds during the 8 years studied, which could be due to the specific morphological shape of these wetlands (12). The highest number of aquatic migratory birds belonged to the duckweed and on average $65.99 \%$ of the total migratory bird population belonged to this species, especially the ducks. Khutka, duckling, lush, and duckling were the most abundant among the migratory aquatic birds in the Anzali Wetland all year (Hossein Jani, Ahmad Nejad and Mehdi Zadeh, 2016). External studies of the diversity and abundance of aquatic birds include Elemberg 1994 studies in Finland and Swedish wetlands and Yang and Quan 2002 in China (Quan, Wen and Yang, 2002). Based on the results of this study, during the study period (2019), 19 species of aquatic and wader birds were observed and identified in Anzali wetland, which were more than Alagol, Almagol and Ajigol wetlands. The size and diversity of aquatic habitats play an important role in attracting aquatic birds and overwintering with regard to their feeding habits. Because habitat is a very good indicator of the nutritional status of different bird species and can greatly affect the population and diversity of birds. Waders are usually found in wet and shallow areas on the 
margins of the wetlands so that they can easily access food and water around their margins and therefore have narrower habitats. Therefore, it can be concluded that the studied wetlands are more favorable for aquatic species. This is consistent with the 2008 results of Behroozirad in the southern margin of the Caspian Sea (Behroozirad, 1999). According to the island's biogeographic theory, and based on Brown and Dinsmore studies, the size of the wetland is the main determinant of species richness and abundance (Brown and Dinsmore, 1986). Therefore, given that Anzali wetland is much larger than other wetlands, its species richness is expected to be higher than other wetlands. However, according to the results of this study, it was shown that the density and diversity of aquatic and wader birds have decreased due to the decrease in the size of the studied wetlands and the changing use of the wetlands. The introduction of nutrients into the wetland and its very good thermal and humidity conditions have led to the rapid growth of plants in the wetland. In recent years, the influx of Azolla, straw and tiffany plants have caused a number of problems, including depletion of dissolved oxygen, blocking the penetration of light spectra to the bottom of the wetland and threatening aquatic life as well as migratory birds.

\section{References}

Amat JA, Green, AJ. Waterbirds as bioindicators of environmental conditions. InConservation monitoring in freshwater habitats 2010 (pp. 45-52). Springer, Dordrecht

Ballan LV, Ghasaian MG, Adamin MS, Klemjir D. Chang in the waterbirds community of the lake seven lake Gilliarea. Biolgy Conservtion. 2002;106:157-63.

BehrooziRad, B. Wetlands in the 21st Century. Journal of Environmental Science. 1999. in Persian.

Brown M, Dinsmore JJ. Implications of marsh size and isolation for marsh bird management. The Journal of Wildlife Management. 1986 Jul 1:392-7.

Brown M, Dinsmore JJ. Implications of marsh size and isolation for marsh bird management. The Journal of Wildlife Management. 1986 Jul 1:392-7. 
Camargo JA. Must dominance increase with the number of subordinate species in competitive interactions?. J Theor Biol. 1993;161:537-42.

Gallego FJ. Remote sensing and land cover area estimation. International Journal of Remote Sensing. 2004 Aug 1;25(15):3019-47.

Ghorbani,R . Taghipour,A . And Mahmoudzadeh, h. Evaluation and Analysis of Land Use Changes in the Alagol, Almagol and Ajigol TurkmanSahara International Wetlands Using Multi-Time Satellite Images. Geography and Environmental Planning. 2012. Page 167. in Persian.

Hossein Jani, Ahmad Nejad, Mehdi Zadeh. Investigation of aquatic plant biomass and their relationship with environmental factors in West Anzali. Wetland Ecobiology Quarterly. 2016. in Persian.

Hosseini Mousavi, S. Trends of Changes in Diversity and Density Indices of Aquatic Birds and Overwintering of Hoorazim and Shadegan Wetlands.
Journal of Wetland Research 12. 2012. in Persian.

Morisita M. Measuring of interspecific association and similarity between communities. Mem. Fac. Sci. Kyushu Univ. Series E. 1959;3:65-80.

Ozesmi SL, Bauer ME. Satellite remote sensing of wetlands. Wetlands ecology and management. 2002 Oct 1;10(5):381402.

Quan RC, Wen X, Yang X. Effects of human activities on migratory waterbirds at Lashihai Lake, China. Biological Conservation. 2002 Dec 1;108(3):273-9.

Riazi,B. Armandi,A. Wintering aquatic birds in Guilan, Mazandaran and Golestan Wetlands and Ibn Wetlands Value Classification by Bird Criteria. Journal of Environmental Studies. 2008. Pages 89. in Persian.

Seaby R, Henderson P. Community Analysis Package 4.0: searching for structure in community data. PISCES Conservation Ltd. Lymington, England. 2007. 
Periódico do Núcleo de Estudos e Pesquisas sobre Gênero e Direito

Centro de Ciências Jurídicas - Universidade Federal da Paraíba V. 9 - $\mathrm{N}^{\circ} 02$ - Ano 2020

ISSN | 2179-7137 | http://periodicos.ufpb.br/ojs2/index.php/ged/index

Simpson EH. Measurement of diversity.

Nature. 1949 Apr;163(4148):688 